\title{
WATER ENTRY OF A FINITE WIDTH WEDGE NEAR A FLOATING BODY
}

C. M. $\mathrm{Bao}^{1}, \mathrm{G} . \mathrm{X} \cdot \mathrm{Wu}^{* 1,2}, \mathrm{G} . \mathrm{Xu}^{1}$

1. School of Naval Architecture and Ocean Engineering, Jiangsu University of Science and Technology, Zhenjiang 212003, P. R. China;

2. Department of Mechanical Engineering, University College London, London WC1E 7JE, UK;

\begin{abstract}
The problem of a two-dimensional finite-width wedge entering water near a freely floating body is considered through the velocity potential theory for the incompressible liquid with the fully nonlinear boundary conditions on the free surface. The problem is solved by using the boundary element method in the time domain. The numerical process is divided into two phases based on whether the interaction between the wedge and floating body is significant. In the first phase, when the single wedge enters water at initial stage, only a small part near its tip is in the fluid, the problem is studied in a stretched coordinate system and the presence of the floating body has no major effect. In the second phase, the disturbance by water entry of the wedge has reached the floating body, and both are considered together in the physical system. The auxiliary function method is adopted to decouple the nonlinear mutual dependence between the motions of the wedge and floating body, both in three degrees of freedom, and the fluid flow, as well as the interaction effects between them. Case studies are undertaken for a wedge entering water in forced or free fall motion, vertically or obliquely. Results are provided for the accelerations, velocities, pressure distribution and free surface deformation, and the interaction effects are discussed.
\end{abstract}

Keywords: Wedge entry near a floating body; Boundary element method; Coupled motion of multi-bodies; Auxiliary function.

\section{Introduction}

In naval architecture and ocean engineering, several vessels may need to coordinate their operations to complete a task. For example, salvage ships work together to recover a sunken ship, a barge off loads oil from a tanker and takes it into the inland water, and cargos are transferred from a damaged ship. The motions of these vessels greatly affect whether the task can be completed successfully. In poor weather condition, the

*Corresponding author. Tel.: +44 207679 3870; fax: +44 2073880180.

E-mail address: g.wu@ucl.ac.uk (G.X.Wu) 
violent flow caused by slamming of a vessel may significantly affect the motion of other vessels. Similar example occurs when a planing hull passes by a small boat. The present work tries to shed some light on this type of problem by considering the mutual interactions when a wedge entering water near a floating body.

Slamming through water entry has been an important topic in naval architecture and ocean engineering. A wedge has been commonly used for case studies. Based on the velocity potential theory with the fully nonlinear boundary conditions, when the effects of gravity and surface tension are ignored, the flow is selfsimilar for constant speed entry, if the wedge is infinite or before the flow along the wedge side exceeds its knuckle. Through conformal mapping, Dobrovol'skaya [1] converted the whole problem into an integral equation for a function along a line. Zhao \& Faltinsen [2] used the numerical method based on the boundary element method. Instead of entry at constant or prescribed speed, $\boldsymbol{W u}$ et al. [3] considered vertical water entry in free fall. Based on their work, $\boldsymbol{X u}$ et al. [4] simulated an asymmetric wedge of three degrees of freedom entering water obliquely. Other work includes that by Semenov \& Iafrati [5] using integral hodograph method for wedge at constant speed.

In above studies, the fluid is always attached on the body surface. In practical problems, the flow may detach from the body as time progresses as a practical structure is always finite. The detachment of flow from the body will have significant effect on the pressure distribution as well as the free surface shape. Zhao et al. [6] studied a finite-width symmetric wedge entering water vertically, and flow detachment from knuckle was modelled. Iafrati \& Battistin [7] studied a similar problem, and a treatment for the jet was applied. Sun \& Faltinsen [8]simulated vertical water entry of an asymmetric bow-shaped structure falling into water, but the motion of the wedge was limited to one degree of freedom. Wang \& Faltinsen [9] investigated vertical water entry of a symmetrical wedge in freefall numerically and experimentally. The open cavity formed after the wedge moved into water was also analyzed. Bao et al. [10] considered a finite-width asymmetric wedge entering water obliquely in forced motion. The gravity is included and an analytical method for the jet flow was introduced based on the momentum equation. Bao et al. [11] further considered a more general water entry problem of a finite-width wedge, in which the wedge was in free fall with three degrees of freedom.

The above works focused on a single body. When a wedge enters water, it will cause large free surface motion nearby. If another structure is nearby, some strong interaction is expected. A typical example is the interactions between the twin hulls of a catamaran or between the main hull and side hulls of trimaran. Wedges are often used again for case studies. $\boldsymbol{W} \boldsymbol{u}[12]$ considered twin wedges entering water, and a three- 
stage method was used. $\boldsymbol{X u}$ et al. [13] investigated twin wedges vertically entering water in freefall. By using OpenFOAM open source, Maki et al. [14] simulated a symmetrical wedge entering water in a constant speed with influence of a single floating ice.

It seems there has been little work on the water entry of a body in free fall near a floating body. The practical relevance of the problem has been highlighted in the first paragraph and it will be the focus of the present work. One of the new challenges is that there will be relative motions between the bodies while they have their own translational and rotational motions. The accelerations of the bodies are unknown. They need to be determined through the hydrodynamic force which is a function of the accelerations. In addition, these accelerations are coupled among themselves. All these mutual and nonlinear dependence is decoupled by adopting the auxiliary method ( $\boldsymbol{W} \boldsymbol{u} \&$ Eatock Taylor [15]) for the two-body problem. This allows all the acceleration components to be found simultaneously.

In the following sections, we shall first introduce the mathematical model and numerical procedure. Then we discuss the auxiliary function method from $\boldsymbol{W u} \boldsymbol{\&}$ Eatock Taylor [15] for the double bodies. Extensive case studies are then undertaken to show the hydrodynamic behaviour of the wedge and the floating body, both of which move in three degrees of freedom, as well as the corresponding pressure distribution and free surface deformation.

\section{Mathematical model and numerical procedure}

\subsection{Governing equation and boundary conditions}

A body is initially freely floating on calm water surface. A wedge of finite height enters water on the right-hand side of the floating-body through free fall motion in three degrees of freedom, as shown in Fig.1. A Cartesian coordinate system $O-x y$ fixed in the space is defined, in which $x$-axis is along the undisturbed water surface and $y$-axis is vertically upward. $i$ and $\boldsymbol{j}$ are the unit vectors in the $x$ and $y$ directions respectively, and $\boldsymbol{k}=\boldsymbol{i} \times \boldsymbol{j}$. At $t=0$, the tip of the wedge touches the calm free surface at the origin $O$. The rotating centres of the wedge and the floating body are located at their centres of the gravity $G_{W}$ and $G_{F}$, respectively, and their rotational velocity are respectively $\boldsymbol{\Omega}_{W}=\omega_{W} \boldsymbol{k}$ and $\boldsymbol{\Omega}_{F}=\omega_{F} \boldsymbol{k}$. The translational velocity of the wedge at point $G_{W}$ is $\boldsymbol{U}_{\boldsymbol{W}}=U_{W} \boldsymbol{i}-V_{W \boldsymbol{j}}$, and the translational velocity of the floating body at point $G_{F}$ is $\boldsymbol{U}_{\boldsymbol{F}}=U_{F} \boldsymbol{i}-V_{F} \boldsymbol{j}$. Here the minus sign before the terms of $\boldsymbol{j}$ means that the vertical velocity is positive when the body moves downwards. 
The water density $\rho$, the vertical velocity $V_{W}$ and the breadth $B_{W}$ of the wedge are used for the nondimensionalisation.

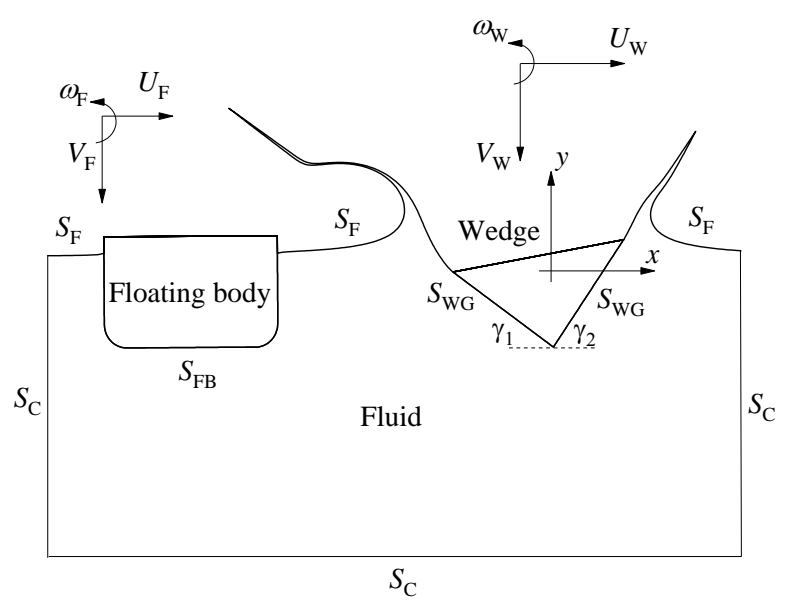

Fig.1 Sketch of the problem

The fluid is assumed to be incompressible and inviscid, and the flow to be irrotational. A velocity potential $\phi$ can then be introduced, which satisfies Laplace equation

$$
\nabla^{2} \phi=0
$$

in the fluid domain. On the wedge surface $S_{W G}$ and the floating body surface $S_{F B}$, we have from the impermeable condition, respectively

$$
\begin{aligned}
& \frac{\partial \phi}{\partial n}=\left(\boldsymbol{U}_{W}+\boldsymbol{\Omega}_{W} \times \boldsymbol{X}_{W}\right) \bullet \boldsymbol{n}=\left(U_{W}-\omega_{W} Y_{W}\right) n_{x}+\left(-V_{W}+\omega_{W} X_{W}\right) n_{y} \\
& \frac{\partial \phi}{\partial n}=\left(\boldsymbol{U}_{F}+\boldsymbol{\Omega}_{F} \times X_{F}\right) \bullet \boldsymbol{n}=\left(U_{F}-\omega_{F} Y_{F}\right) n_{x}+\left(-V_{F}+\omega_{F} X_{F}\right) n_{y}
\end{aligned}
$$

where $\boldsymbol{n}=\left(n_{x}, n_{y}\right)$ is the normal vector of the body surface pointing out of the fluid domain. $\boldsymbol{X}_{W}=\left(X_{W}, Y_{W}\right)$ and $\boldsymbol{X}_{\boldsymbol{F}}=\left(X_{F}, Y_{F}\right)$ are the position vectors relative to each corresponding centre of rotation. The Lagrangian form of the kinematic and dynamic conditions on the free surface $S_{F}$ can be written as

$$
\begin{aligned}
& \frac{D x}{D t}=\frac{\partial \phi}{\partial x}, \frac{D y}{D t}=\frac{\partial \phi}{\partial y} \\
& \frac{D \phi}{D t}=-\frac{y}{F r^{2}}+\frac{1}{2}|\nabla \phi|^{2}
\end{aligned}
$$

where $F r=V_{W} / \sqrt{g B_{W}}$ is the Froude number. The atmospheric pressure on the water surface has been assumed to be constant in Eq.(5). In addition, we specify a far-field condition

$$
\frac{\partial \phi}{\partial n}=0, \quad \sqrt{x^{2}+y^{2}} \rightarrow \infty
$$

on the basis that the fluid is undisturbed far away from the body. 
When the wedge enters water, it touches the fluid through a single point. The fluid region disturbed at early stage of the water entry is confined near the body. To capture the local flow inside the disturbed region, the element size must be very small. If the wedge and the floating body are considered together at this stage, the number of the element will be exceedingly large. Here we adopt the method by $\boldsymbol{W u}$ [12]. Water entry with influence on a floating body can be divided into two phases to simulate. In the first phase, a single wedge is considered in the domain in Fig.2, as the interaction with the floating body is negligible at this stage. The stretched coordinate system (Wu et al. [3]) can be used. We define

$$
\phi(x, y, t)=s(t) \varphi(\alpha, \beta, t), \alpha=x / s(t), \beta=y / s(t)
$$

where $s(t)$ is the vertical displacement of the centre $G_{W}$ of rotation of the wedge:

$$
s(t)=\int_{0}^{t} V_{\mathrm{w}}(\tau) d \tau
$$

In the stretched coordinate system, the free surface boundary condition can be written as

$$
\begin{aligned}
& \frac{D(s \alpha)}{D t}=\frac{\partial \varphi}{\partial \alpha}, \frac{D(s \beta)}{D t}=\frac{\partial \varphi}{\partial \beta} \\
& \frac{D(s \varphi)}{D t}=-\frac{s \beta}{F r^{2}}+\frac{1}{2}\left(\varphi_{\alpha}^{2}+\varphi_{\beta}^{2}\right)
\end{aligned}
$$

The use of the stretched coordinate system is particularly effective when $s$ is small. When $s$ has reached a finite value, the simulation can continue in the stretched coordinate system or move back to the physical system $O-x y$. During the process, the interaction with the nearby floating body can be considered when its effect is no longer negligible. The simulation moves to the second phase, in which both the wedge and the floating body are included simultaneously in the computational domain as shown in Fig.1.

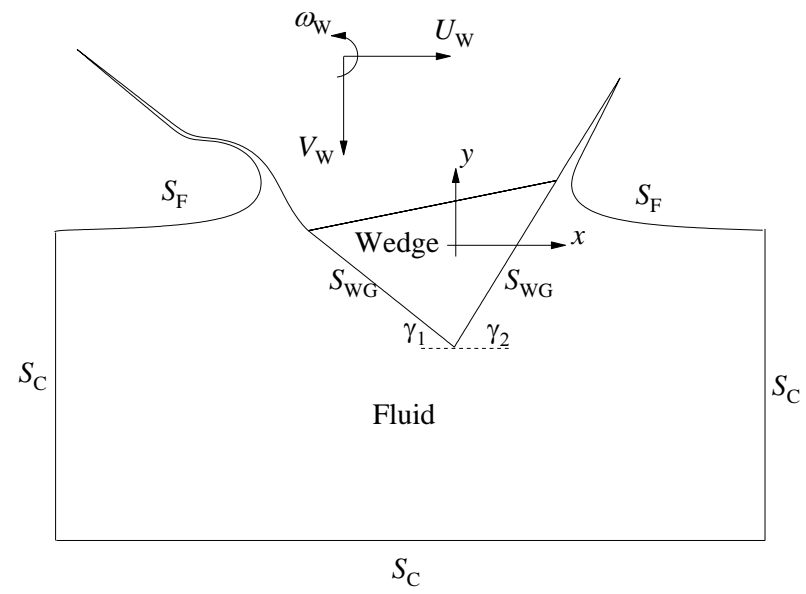

Fig.2 Computational domain for a single wedge. 


\subsection{Boundary integral equation}

To solve the boundary value problem in Sect. 2.1, we transform the differential equation in the fluid domain into the following boundary integral equation based on Green's second theorem.

$$
A(p) \varphi(p)=\int_{S}\left[\ln r_{p q} \frac{\partial \varphi(q)}{\partial n_{q}}-\varphi(q) \frac{\partial}{\partial n_{q}}\left(\ln r_{p q}\right)\right] d S_{q}
$$

where $A(p)$ is the solid angle of point $p$ on the fluid boundary, and $r_{p q}$ is the distance between points $p$ and $q$. $S$ in Eq.(11) includes all the surfaces in Fig.2 at stage 1 and all the surfaces in Fig.1 at stage 2. It is discretized into many small elements. Within each element linear variation with local coordinate $\xi$

$$
f=\sum_{i=1}^{2} N_{i}(\xi) f_{i}=N_{1}(\xi) f_{1}+N_{2}(\xi) f_{2}
$$

is assumed, where

$$
N_{1}(\xi)=1-\xi, N_{2}(\xi)=\xi
$$

$f$ in Eq. (12) stands for $\varphi$ or $\varphi_{n}$, and the subscripts 1 and 2 correspond to the two nodes of the element, where the local coordinate $\xi$ is 0 and 1 respectively. Thus Eq.(11) can then be written as

$$
A(p) \varphi(p)+\sum_{k=1}^{N_{e}} \sum_{i=1}^{2} \varphi_{k}^{i}(q) \int_{0}^{1} \frac{\partial}{\partial n_{q}}\left(\ln r_{p q}\right) N_{i}(\xi) l_{k} d \xi=\sum_{k=1}^{N_{e}} \sum_{i=1}^{2} \frac{\partial \varphi_{k}^{i}(q)}{\partial n_{q}} \int_{0}^{1} \ln r_{p q} N_{i}(\xi) l_{k} d \xi(14)
$$

where $i=1,2$ respectively denote the first and second nodes of the $k$ th element with length $l_{k}$, and $N_{e}$ is the total number of elements. With this notation, we have $\varphi_{k-1}^{2}=\varphi_{k}^{1}=\varphi_{k}$. By letting point $p$ approach each element node, a system of equations can be obtained.

$$
[H]\{\varphi\}=[G]\left\{\varphi_{n}\right\}
$$

where the matrices $[\mathrm{H}]$ and $[\mathrm{G}]$ contain the integrals of $\partial\left(\ln r_{p q}\right) / \partial n_{q}$ and $\ln r_{p q}$ over each element $(\boldsymbol{L u}$ et al. [16]), respectively, and $\{\varphi\}$ and $\left\{\varphi_{n}\right\}$ are columns containing the potentials $\varphi_{k}$ and its normal derivatives $\varphi_{n k}$ on all the element nodes.

At each time step, $\varphi$ on the free surface and $\varphi_{n}$ on the solid body surface are known, both of which can be moved to the right-hand side of the equation. The unknown can be moved to the left-hand side. Subsequently, Eq.(15) can be rearranged as follow 


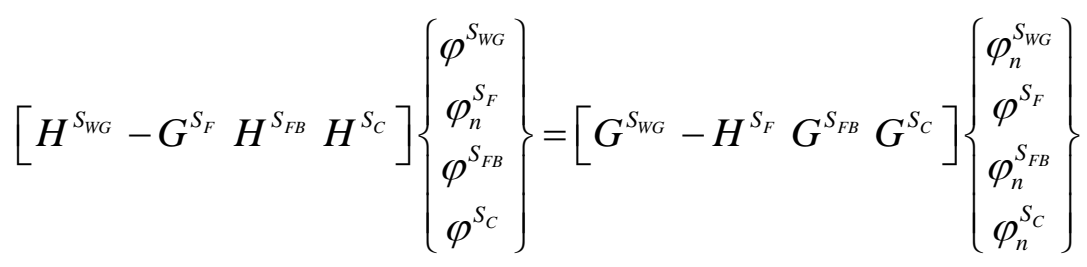

where the superscripts correspond to the surfaces defined in Fig.1. During the first stage, all the terms of $S_{F B}$ are removed from the matrix equation. Once the solution of Eq.(16) at each time step has been obtained, we can update the free surface profile through the kinematic boundary conditions given by Eq.(4) while the dynamic boundary condition in Eq.(5) is used to update the velocity potential on the free surface.

When a wedge enters water, a long and thin jet is usually formed on the surface of the wedge. That is always problematic in numerical simulation. In the present paper, the jet flow is kept. Before flow detachment, the jet is attached on the surface of the wedge. The treatment for the jet is similar to that proposed by $\boldsymbol{W u}$ [17] When the flow is detached from the knuckle, both of its sides become free surface and we adopt the method proposed by Bao et al. [10].

At the intersection of the free surface and the body surface, we assume that the flow leaves the knuckle tangentially (Bao et al. [11]), $\phi_{n}$ is therefore continuous at the knuckle and is known from the body surface boundary condition. The velocity potential at the intersection after detachment can be treated as unknown and can be obtained by solving Eq.(16).

\subsection{Equations for motions for two freely floating bodies with coupling effects}

In the first stage, water entry of a finite-width wedge is considered in isolation. The mutual dependence of the body motion and the fluid flow is decoupled by using the auxiliary function method proposed by $\boldsymbol{W u}$ \& Eatock Taylor [15], as has been done by Bao et al. [11]. Here we apply the auxiliary function method to two freely floating bodies whose interactions need to be accounted for at the second stage. From Newton's second law, the equations of motions for the wedge and the floating body can be respectively written as:

$$
\begin{aligned}
& {\left[M_{W}\right]\left[\dot{U}_{W}\right]=\left[F_{W h}\right]+\left[F_{W e}\right]} \\
& {\left[M_{F}\right]\left[\dot{U}_{F}\right]=\left[F_{F h}\right]+\left[F_{F e}\right]}
\end{aligned}
$$

where $\left[M_{\mathrm{w}}\right]$ and $\left[M_{F}\right]$ are respectively the mass matrices of the wedge and the floating body, $\left[\dot{U}_{\mathrm{w}}\right]$ and $\left[\dot{U}_{F}\right]$ are respectively the columns of their horizontal, vertical and rotational accelerations, $\left[F_{\mathrm{w} h}\right]$ and $\left[F_{F h}\right]$ are respectively the columns of hydrodynamic forces and moments on the two bodies, and $\left[F_{w e}\right]$ and $\left[F_{F e}\right]$ are 
the corresponding external forces and moments.

The velocity potential can be obtained after the boundary integral equation is solved. The pressure on body surface can be obtained by the Bernoulli equation

$$
p=-\left(\phi_{t}+\frac{1}{2} \nabla \phi \nabla \phi+\frac{y}{F r^{2}}\right)
$$

The force and the moment on the wedge and the floating body can be obtained respectively by the integral the pressure along the wetted surface of the wedge and the floating body.

$$
\begin{aligned}
& \boldsymbol{F}_{W}=\int_{S_{W G}} p \boldsymbol{n} d S \\
& \boldsymbol{M}_{W}=\int_{S_{W G}} p(\boldsymbol{X} \times \boldsymbol{n}) d S \\
& \boldsymbol{F}_{F}=\int_{S_{F B}} p \boldsymbol{n} d S \\
& \boldsymbol{M}_{F}=\int_{S_{F B}} p(\boldsymbol{X} \times \boldsymbol{n}) d S
\end{aligned}
$$

However, $\phi_{t}$ in Eq.(19) is still not explicitly known even though $\phi$ has been found. Here we notice that $\phi_{t}$ in fluid domain satisfies the Laplace equation.

$$
\nabla \phi_{t}=0
$$

The body surface boundary condition on the wedge surface can be written as ( $\boldsymbol{W} \boldsymbol{u}[18])$ :

$$
\frac{\partial \phi_{t}}{\partial n}=\left(\dot{\boldsymbol{U}}_{W}+\dot{\boldsymbol{\Omega}}_{W} \times \boldsymbol{X}_{W}\right) \cdot \boldsymbol{n}-\boldsymbol{U}_{W} \cdot \frac{\partial \nabla \phi}{\partial n}+\boldsymbol{\Omega}_{W} \cdot \frac{\partial}{\partial n}\left[\boldsymbol{X}_{W} \times\left(\boldsymbol{U}_{W}-\nabla \phi\right)\right]
$$

and on the floating body surface as:

$$
\frac{\partial \phi_{t}}{\partial n}=\left(\dot{\boldsymbol{U}}_{F}+\dot{\boldsymbol{\Omega}}_{F} \times \boldsymbol{X}_{F}\right) \cdot \boldsymbol{n}-\boldsymbol{U}_{F} \cdot \frac{\partial \nabla \phi}{\partial n}+\boldsymbol{\Omega}_{F} \cdot \frac{\partial}{\partial n}\left[\boldsymbol{X}_{F} \times\left(\boldsymbol{U}_{F}-\nabla \phi\right)\right]
$$

$\phi_{t}$ on the free surface can be obtained by letting Eq.(19) be equal to zero:

$$
\phi_{t}=-\frac{1}{2} \nabla \phi \nabla \phi-\frac{y}{F r^{2}}
$$

The problem for $\phi_{t}$ might be solved in a manner similar to that used for $\phi$ itself if the accelerations of the bodies were known. However, these accelerations are yet to be found, which depended on $\phi_{t}$ as shown in Eqs.(17) to (23). To decompose their mutual dependence, we introduce some auxiliary functions $\chi$ and $\lambda_{i}$ $(i=1 \sim 6)$, which satisfy Laplace equation, and write $\phi_{t}$ as

$$
\phi_{t}=\chi+\dot{U}_{1} \lambda_{1}+\left(-\dot{U}_{2}\right) \lambda_{2}+\dot{U}_{3} \lambda_{3}+\dot{U}_{4} \lambda_{4}+\left(-\dot{U}_{5}\right) \lambda_{5}+\dot{U}_{6} \lambda_{6}
$$


The boundary conditions for $\chi$ and $\lambda_{i}(i=1 \sim 6)$ can be given based on those for $\phi_{t}$. On the wedge surface

$$
\begin{aligned}
& \frac{\partial \chi}{\partial n}=-\boldsymbol{U}_{W} \cdot \frac{\partial \nabla \phi}{\partial n}+\boldsymbol{\Omega}_{W} \cdot \frac{\partial}{\partial n}\left[\boldsymbol{X}_{W} \times\left(\boldsymbol{U}_{W}-\nabla \phi\right)\right] \\
& \frac{\partial \lambda_{i}}{\partial n}=n_{i} \quad(i=1 \sim 3) \\
& \frac{\partial \lambda_{i}}{\partial n}=0 \quad(i=4 \sim 6)
\end{aligned}
$$

On the free surface

$$
\begin{aligned}
& \chi=-\frac{1}{2} \nabla \phi \nabla \phi-\frac{y}{F r^{2}} \\
& \lambda_{i}=0 \quad(i=1 \sim 6)
\end{aligned}
$$

On the floating body surface

$$
\begin{aligned}
& \frac{\partial \chi}{\partial n}=-\boldsymbol{U}_{F} \cdot \frac{\partial \nabla \phi}{\partial n}+\boldsymbol{\Omega}_{F} \cdot \frac{\partial}{\partial n}\left[\boldsymbol{X}_{F} \times\left(\boldsymbol{U}_{F}-\nabla \phi\right)\right] \\
& \frac{\partial \lambda_{i}}{\partial n}=0 \quad(i=1 \sim 3) \\
& \frac{\partial \lambda_{i}}{\partial n}=n_{i} \quad(i=4 \sim 6)
\end{aligned}
$$

On the control surface

$$
\begin{aligned}
& \frac{\partial \chi}{\partial n}=0 \\
& \frac{\partial \lambda_{i}}{\partial n}=0 \quad(i=1 \sim 6)
\end{aligned}
$$

where $\left(n_{1}, n_{2}, n_{3}\right)=\left(n_{x}, n_{y}, X_{\mathrm{w}} n_{y}-Y_{\mathrm{w}} n_{x}\right)$ on the wedge, $\left(n_{4}, n_{5}, n_{6}\right)=\left(n_{x}, n_{y}, X_{F} n_{y}-Y_{F} n_{x}\right)$ on the floating body. Compared with the method for a single, we noticed there are six auxiliary functions $\lambda_{i}(i=1 \sim 6)$ instead of three.

The boundary value problems for $\chi$ and $\lambda_{i}(i=1 \sim 6)$ can be solved in the same way as $\phi$. It should be noted that Eq.(29) and(34) contain a second derivative which is usually difficult in numerical calculation to ensure sufficient accuracy. We adopt the method by $\boldsymbol{X} \boldsymbol{u} \boldsymbol{\&} \boldsymbol{W} \boldsymbol{u}[19]$ to deal with this second derivative.

$$
\frac{\partial \phi_{y}}{\partial n}=\frac{\partial \phi_{x}}{\partial l}, \frac{\partial \phi_{x}}{\partial n}=-\frac{\partial \phi_{y}}{\partial l}
$$

where $l$ is the tangential vector of the body surface.

Substituting $\phi_{t}$ in Eq.(28) with the auxiliary functions $\chi$ and $\lambda_{i}(i=1 \sim 6)$ into Eq.(17) to Eq.(23), the 
following formula can be obtained

$$
\sum_{i=1}^{6}\left(\delta_{i j} M_{i i}+N_{i j}\right) \cdot \dot{U}_{i}=f_{i}-\frac{M_{i i} \delta_{i 2}}{F r^{2}}-\frac{M_{i i} \delta_{i 5}}{F r^{2}}
$$

where

$$
\begin{gathered}
N_{i j}= \begin{cases}\iint_{s_{\mathrm{w}}} \lambda_{i} \bullet n_{j} d S \quad(j=1 \sim 3) \\
\iint_{s_{F B}} \lambda_{i} \bullet n_{j} d S \quad(j=4 \sim 6)\end{cases} \\
f_{i}= \begin{cases}-\iiint_{s_{W}}\left(\chi+\frac{1}{2} \nabla \phi \nabla \phi+\frac{y}{F r^{2}}\right) n_{i} d S \quad(i=1 \sim 3) \\
-\iint_{s_{F B}}\left(\chi+\frac{1}{2} \nabla \phi \nabla \phi+\frac{y}{F r^{2}}\right) n_{i} d S \quad(i=4 \sim 6)\end{cases}
\end{gathered}
$$

and $\delta_{i j}=\left\{\begin{array}{ll}1 & j=i \\ 0 & j \neq i\end{array}\right.$,

$$
\begin{aligned}
& \left(M_{11}, M_{22}, M_{33}\right)=\left(M_{W}, M_{W}, I_{W}\right),\left(M_{44}, M_{55}, M_{66}\right)=\left(M_{F}, M_{F}, I_{F}\right) \\
& \left(\dot{U}_{1}, \dot{U}_{2}, \dot{U}_{3}\right)=\left(\dot{U}_{W},-\dot{V}_{W}, \dot{\omega}_{W}\right),\left(\dot{U}_{4}, \dot{U}_{5}, \dot{U}_{6}\right)=\left(\dot{U}_{F},-\dot{V}_{F}, \dot{\omega}_{F}\right)
\end{aligned}
$$

where $M_{\mathrm{w}}$ and $M_{F}$ are respectively the mass of the wedge and the floating body, and $I_{\mathrm{w}}$ and $I_{F}$ are their rotational inertia respectively. The accelerations of the wedge and the floating body, both in three degrees of freedom, can be obtained directly by Eq.(40).

When a wedge enters water at a constant speed, the acceleration terms in Eq.(25) are zero. Eq.(40) will be a $3 \times 3$ equations for accelerations of the floating body. Those auxiliary functions $\lambda_{i}(i=1 \sim 3)$ are no longer needed. Therefore, $\phi_{t}$ can be written as

$$
\phi_{t}=\chi+\dot{U}_{4} \lambda_{4}+\left(-\dot{U}_{5}\right) \lambda_{5}+\dot{U}_{6} \lambda_{6}
$$

Similarly, if the floating body is fixed and the wedge enters water in freefall, then

$$
\phi_{t}=\chi+\dot{U}_{1} \lambda_{1}+\left(-\dot{U}_{2}\right) \lambda_{2}+\dot{U}_{3} \lambda_{3}
$$

After all the accelerations of the wedge and the floating body are found, all the velocities can be updated, as well as the positions and orientations of both bodies. Therefore, we then can specify the boundary conditions 
on the wedge and the floating body surfaces with the new velocities on their new positions for the next time step.

\section{Numerical results and discussions}

At $t=0$, the tip of the wedge is touching the calm free surface and the contact point is taken as the origin of the system, as shown in Fig. $3 \mathrm{~b}$. Heel angle $\theta_{\mathrm{w}}$ in the figure is the angle between the symmetry line of the wedge and $y$-axis. The wedge may be asymmetric about $y$ axis and has left and right deadrise angles $\gamma_{1}$ and $\gamma_{2}$, respectively. The angle between the symmetry line of the wedge and its face is $\gamma$. These angles form the following relationships:

$$
\gamma_{1}=\frac{\pi}{2}+\theta_{\mathrm{w}}-\gamma, \gamma_{2}=\frac{\pi}{2}-\theta_{\mathrm{w}}-\gamma
$$

The rotating centre of the wedge is taken at centre of the mass $G_{\mathrm{w}}$, which is above the top of the wedge based on the assumption that there may be a structure attached to the wedge in practical problems. The distance between $G_{\mathrm{w}}$ and the tip of the wedge is $l_{\mathrm{w}}$. The breadth of the wedge top is $B_{\mathrm{w}}$.
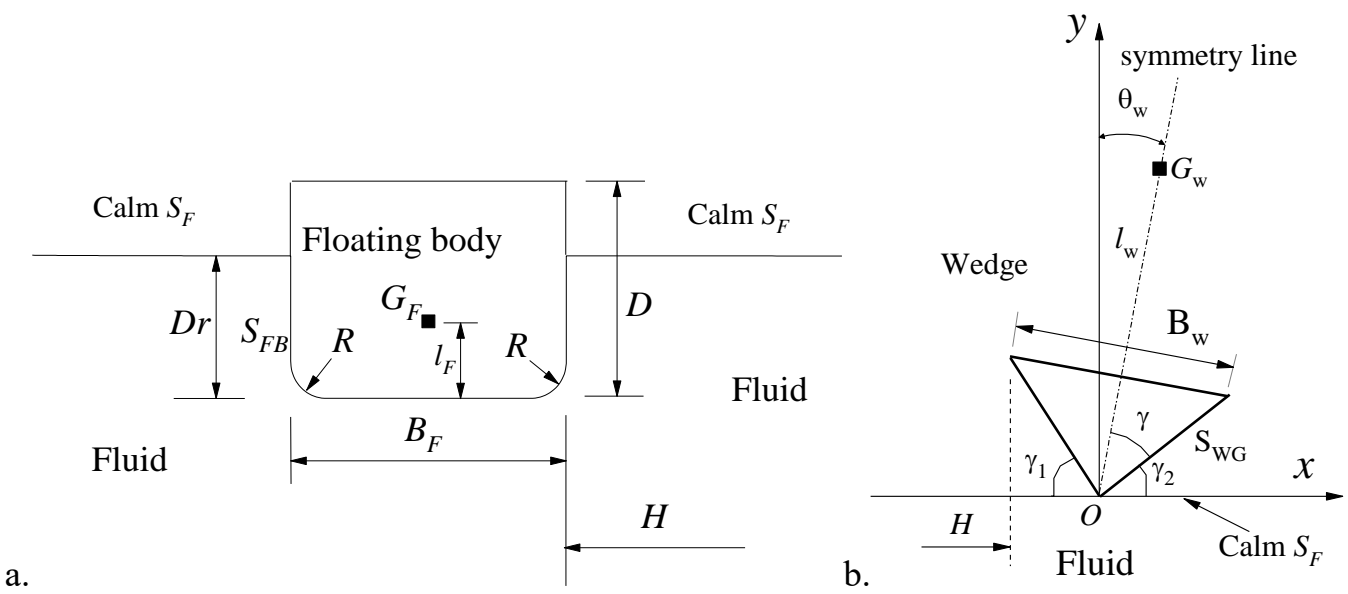

Fig. 3 The parameters, (a) the wedge, (b) the floating body

In Fig.3a, the floating body is initially on the calm free surface on the left-hand side of the wedge. In the cases considered below, the floating body is taken a rectangular. with width of $B_{F}$, height of $D$ and draught of $D r$. The two sharp corners at the bottom are rounded by a quarter of a circle with small radius $R$. The rotational centre is taken at the centre of its mass $G_{F}$, and its distance to the bottom plate is $l_{F}$.

Some nondimensional data of the wedge and the floating body are given in Tab.1, respectively. The wedge model was used in Bao et al. [10] and Bao et al. [11], in which a single finite-width wedge entering water at a constant speed and in freefall was respectively investigated numerically. The floating body model 
has been used to study its motion in waves, by $\mathbf{K o o} \boldsymbol{\&} \boldsymbol{K} \boldsymbol{i m}$ [20], Wang et al. [21]. The floating body initially at rest is on the calm free surface, and its weight is balanced by its buoyancy. Thus, its initial draught $D r$ equals to 0.5 when $M_{F}=0.493$. These data will be used unless specified otherwise.

\begin{tabular}{c|c|c|c|c|c}
\hline$\gamma$ & $M_{\mathrm{w}}$ & $B_{\mathrm{w}}$ & $l_{\mathrm{w}}$ & $I_{\mathrm{w}}$ & $M_{F}$ \\
\hline $45^{\circ}$ & 2.5 & 1 & 1.25 & 28.125 & 0.493 \\
\hline$I_{F}$ & $D$ & $B_{F}$ & $R$ & $l_{F}$ & $D r$ \\
\hline $6.39 \times 10^{-2}$ & 0.6 & 1 & 0.128 & 0.27 & 0.5 \\
\hline
\end{tabular}

Tab.1 The data of the wedge and floating body

\subsection{Convergence study}

At the first stage, the boundary of the fluid domain includes the free surface $S_{F}$, the wedge body surface $S_{W G}$ and the control surface $S_{C}$. At the second stage, it also includes the floating body surface $S_{F B}$. The whole boundary is discretized by using straight elements. Elements of typical length $\Delta l$ are uniformly distributed on the wedge surface $S_{W G}$, the floating body surface $S_{F B}$ and on the free surface $S_{F}$ near these bodies. On the free surface away from the body, the size of the element increases gradually at a fixed ratio, and the largest element far away from the body is no more than five times typical length $\Delta l$. The time step $\Delta t$ is determined by

$$
\Delta t=C \frac{\Delta l}{\max \left(\left|(\nabla \phi)_{\mathrm{SF}}\right|\right)}
$$

where the coefficient $C$ is chosen in such a way to ensure that the fluid particle on the free surface will move only a small fraction of grid size within one-time step, and $0<C<1$. During the simulation, the time step will be adjusted based on the Eq.(48) through the ratio of the smallest element size and the largest velocity magnitude.

We consider an asymmetric wedge with heel angle $\theta_{W 0}=-10^{\circ}$ entering water vertically in freefall while a freely floating body is nearby with the horizontal distance between its starboard and the left knuckle of the wedge $H_{0}=1$. The initial vertical velocity of the wedge is $F r_{0}=3$. Here and in the following simulations, a variable with subscript 0 indicates its initial value. We run simulations for this case by setting different pairs of typical element length $\Delta l$ and coefficient $C$, with $\Delta l=0.02$ and $C=0.2, \Delta l=0.02$ and $C=0.4, \Delta l=0.04$ and $C=0.2$ respectively in the stretched system. While both the wedge and the floating body have three degrees 
of freedom, we provide only part of their results. Fig.4 gives the horizontal accelerations of the floating body $\dot{U}_{F}$ and the wedge $U_{W}$, the free surface profile and the pressure distribution on the floating body surface $P_{F}$ at $t=1$. These results of $\Delta l=0.02, C=0.2$ and $\Delta l=0.04, C=0.2$ are in good agreement. This shows that the present method is already mesh independent. These results of $\Delta l=0.02, C=0.2$ and $\Delta l=0.02, C=0.4$ coincide well, which indicates that the present method has achieved convergence with time step as well. $C$ is taken as 0.4 in the following simulations together with $\Delta l=0.02$.
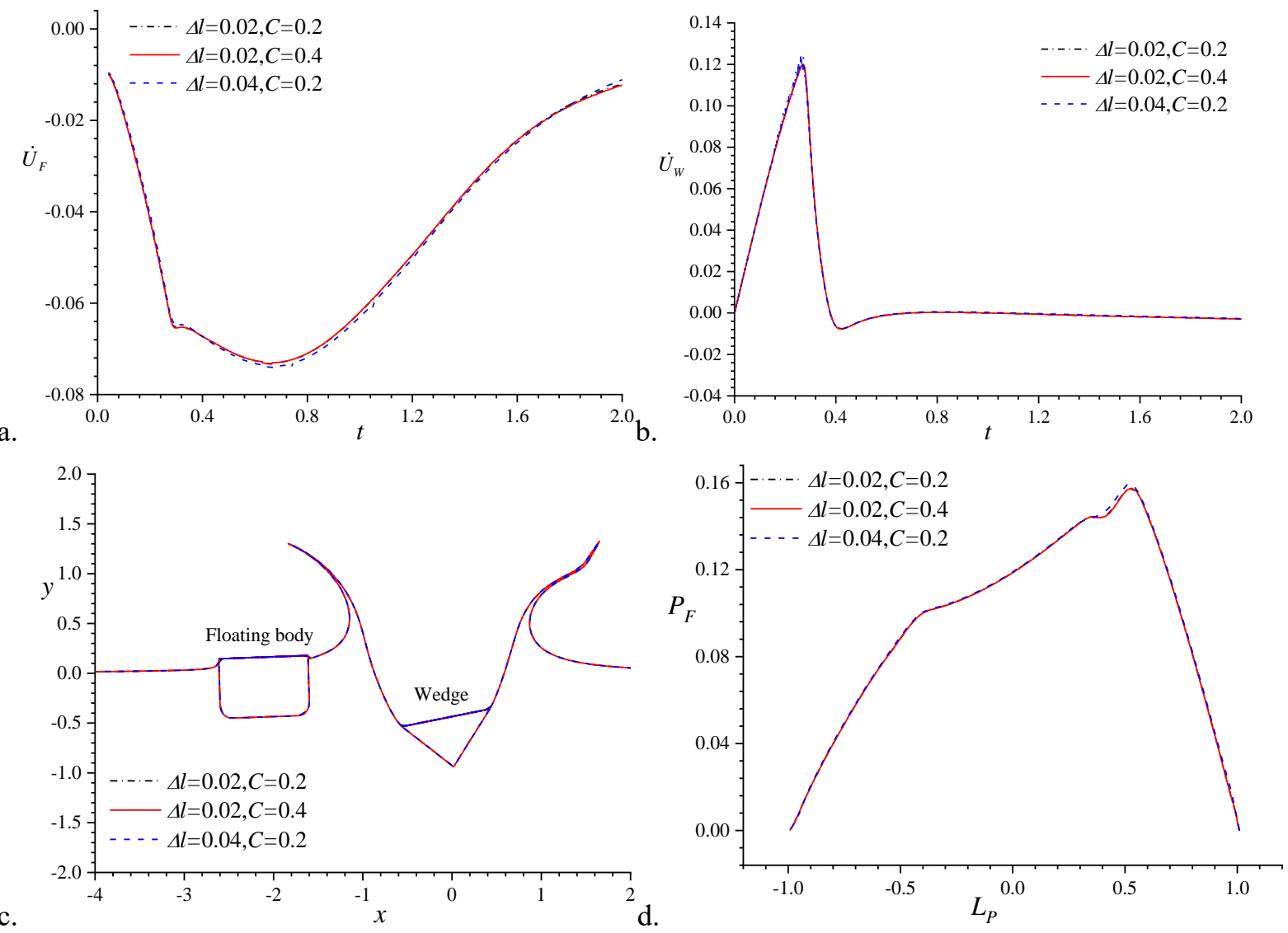

Fig. 4 An asymmetric wedge $\left(\theta{ }_{w 0}=-10^{\circ}\right)$ entering water vertically $\left(F r_{0}=3\right)$ in freefall with different typical element length $\Delta l$ and time step coefficient $C$. (a) horizontal acceleration of the floating body $U_{F}$, (b) horizontal acceleration of the wedge $\hat{U}_{W}$, (c) free surface profile at $t=1$, (d) pressure distribution on the floating body surface $P_{F}$ at $t=1$.

As discussed previously, the present simulation process is divided into two phases. In the first phase, only a single wedge is considered. The computational domain is chosen as a rectangular control box which is truncated at $\alpha= \pm L_{C}$ and $\beta=-40$ in the stretched coordinate system. When the left control surface in the physical coordinate system $s(t) L_{C}$ moves towards the floating body and the interaction between the bodies is no longer insignificant, for example when the control surface in the physic domain is at $x=-0.9 H$, the simulation moves into the second phase, in which both the wedge and the floating body are considered together in physical coordinate system. Hence, the value of $L_{C}$ will affect the time of the transition from the 
first stage to the second. To verify that the control surface is far enough and has little effect on the simulation process, we set $L_{C}=15$, and 25 respectively in the stretched system. The results in Fig.5a show that the starting time of the horizontal acceleration of the floating body is different for different $L_{C}$, at which the simulation turns to the second phase. The starting time $t=0.041$ for $L_{C}=25$ and $t=0.069$ for $L_{C}=25$. However, in Fig.5, those results agree very well. $L_{C}$ is set to 25 in the following simulations
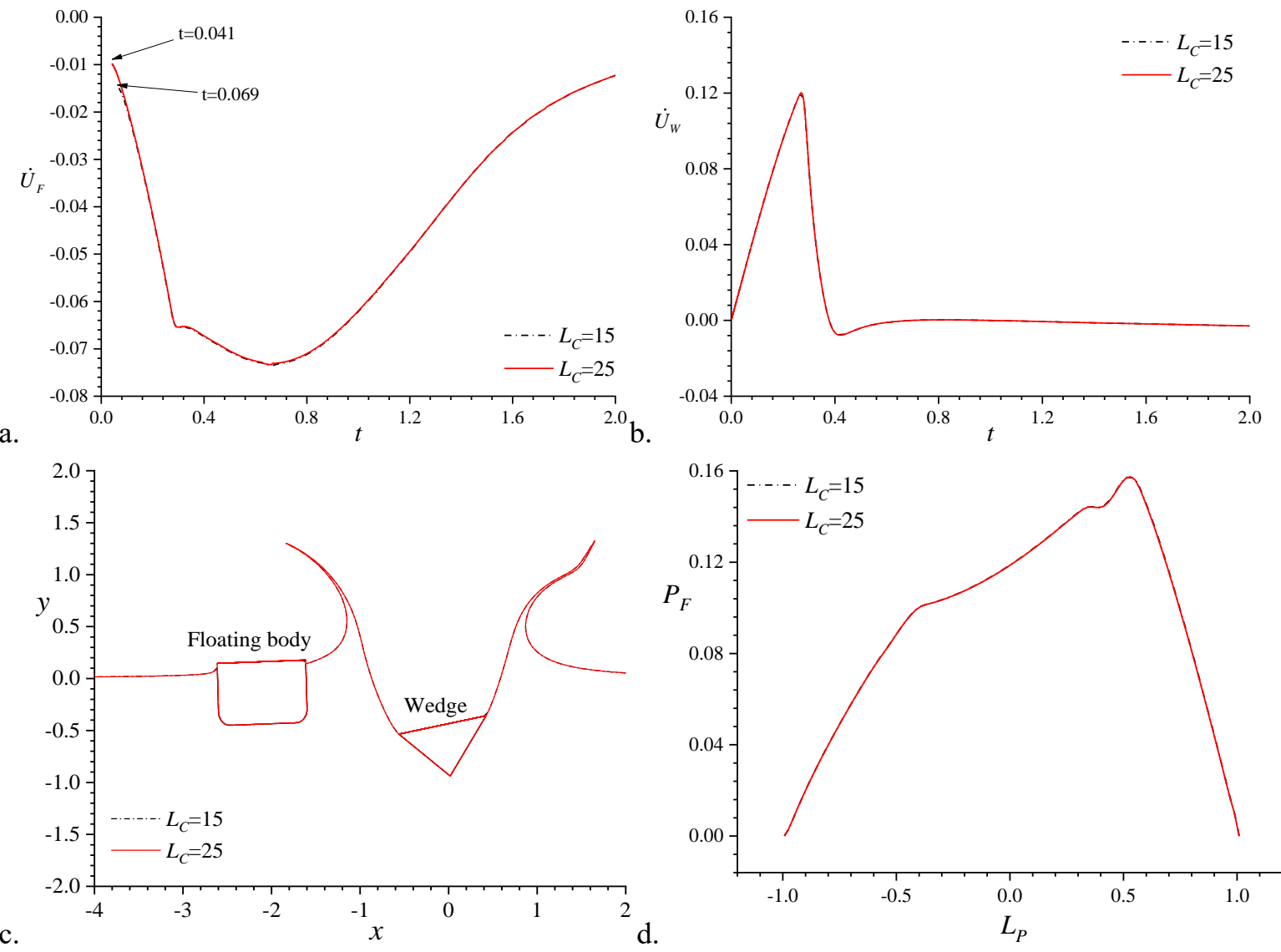

Fig.5 An asymmetric wedge $\left(\theta_{W 0}=10^{\circ}\right)$ entering water vertically $\left(F r_{0}=3\right)$ in freefall with different control surface in the stretched coordinate system. (a) horizontal acceleration of the floating body $\dot{U}_{F}$, (b) horizontal acceleration of the wedge $U_{W}$, (c) free surface profile at $t=1$, (d) pressure distribution on the floating body surface $P_{F}$ at $t=1$.

\subsection{Vertical entry of a symmetrical wedge at a constant speed.}

When a wedge enters water, it may generate violent flow. The fluid region disturbed by the entry will expand with time rapidly. If a floating body is nearby, it will experience the effect very quickly. In turn, flow will change because of the presence of the floating body, including the surrounding free surface, and this will then affect the force on the wedge. We consider a case that a symmetrical wedge with deadrise angle $\gamma_{1}=\gamma_{2}=45^{\circ}$ and width $B_{\mathrm{w}}=1$ vertically enters water at constant speed of $F r=3$. The floating body initially at rest is on the left-hand side of the wedge with $H_{0}=1$ and its weight is balanced by its buoyancy. Fig. 6 shows comparison 
between results for wedge near the floating body and wedge in isolation. Without the floating body, the horizontal force and the moment of the wedge on the single wedge are zero all the time. When the floating body is present, its influence on the wedge is negligible at the initial stage. The influence becomes more evident as time increases. The difference between those results with and without the floating body becomes obvious. For the vertical force, it is mainly dominated by its water entry. The effect of the floating body gradually becomes visible. However, relative to the water entry force itself, it is still small.

a.
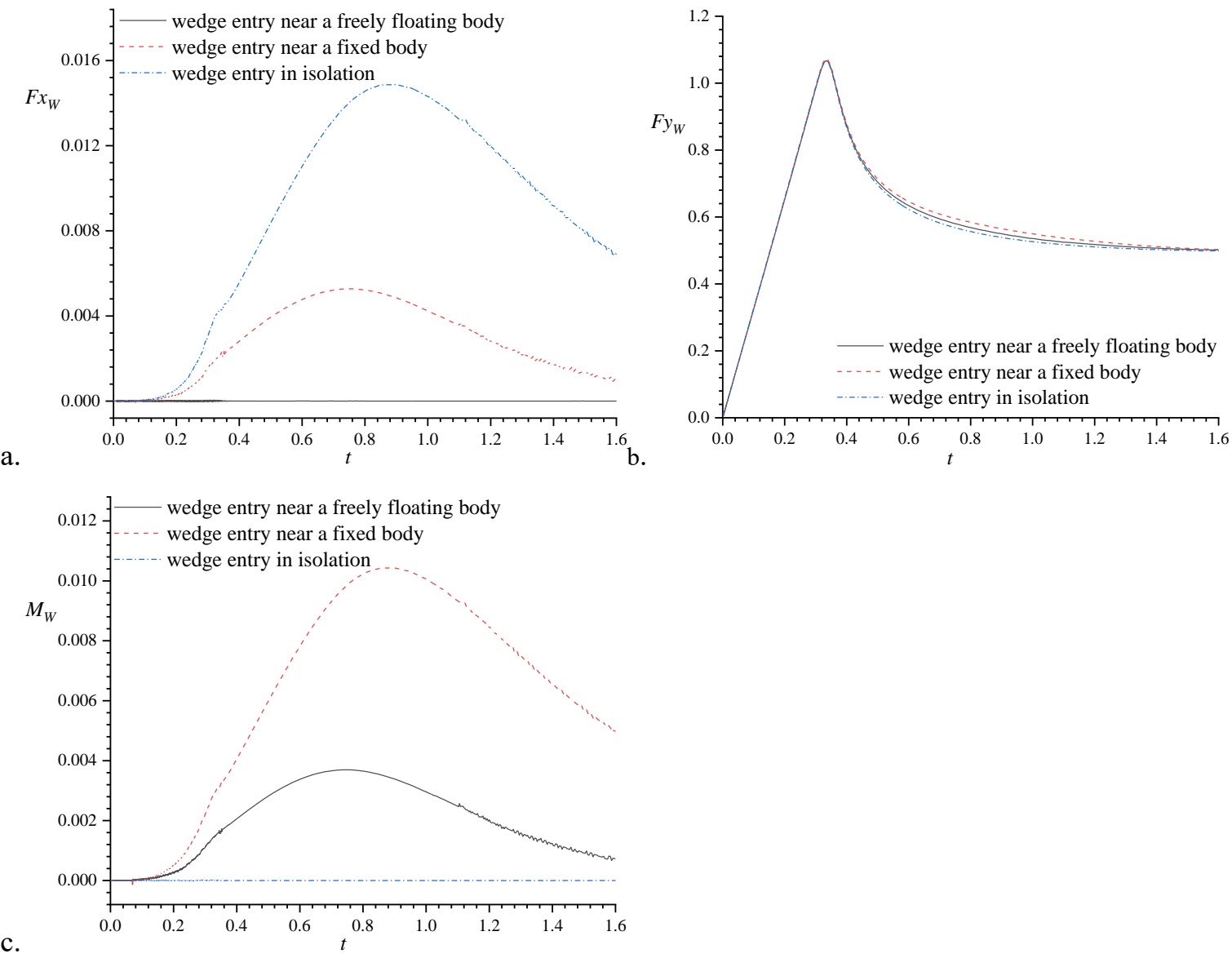

Fig.6 The forces and moment on the wedge entering water vertically near a floating body.

(a) horizontal force, (b) vertical force, (c) moment.

Fig.7 provides the free surface profiles at different time instants. As shown in Fig.7a, the free surface around the floating body is nearly flat at initial stage $t<0.5$. In Fig.7b, at later stager $t \geq 0.8$, the wedge will be fully below the calm water surface. The fluid around the floating body has change significantly, and the free surface runup has passed the knuckle on the right-hand side of the floating body after $t=1.1$. To study the influence of the presence of the floating body, we take the free surface profile at $t=1.7$ from Fig. 7 and put it in Fig. 8 with free surface profiles for a fixed body and a single wedge at the same time. Around the floating body, the free surface will rise up. The runup of the free surface on the body surface can exceed the body 
height and the fluid can depart from the body surface, similar to the detachment from the wedge surface. For a fixed body the detachment occurs earlier. When the body is free to respond, the fluid flow is less blocked by the body surface. The floating body will be pushed to the left and vertically upwards by the fluid force, and rotate anti-clockwise as the centre of rotation is relatively low. The motion of the freely floating body will slow down the runup and the flow will detach from the body later than from the fixed body. Fig.7 also shows that the presence of the body does not have major effect on the free surface near the wedge. In fact, initially at stage one, the wedge is virtually in isolation and the effect of the floating body is negligible. Later on, when the wedge has moved down below the calm water surface, its distance to the floating body increases and the effect of the floating body decreases.

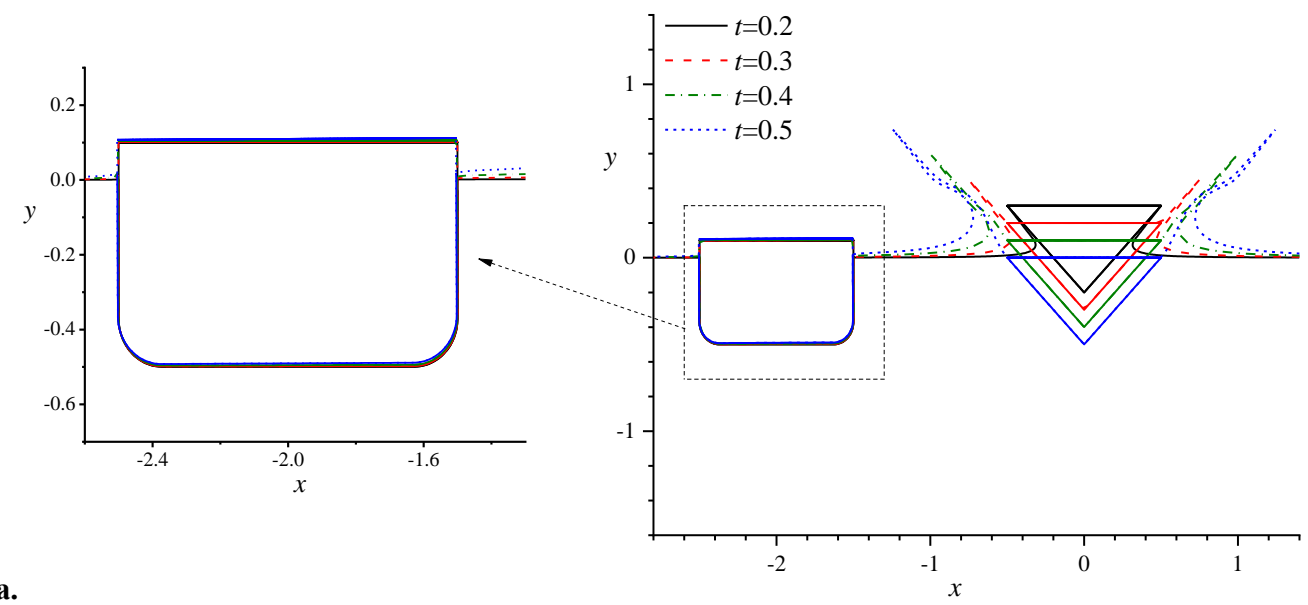

a.

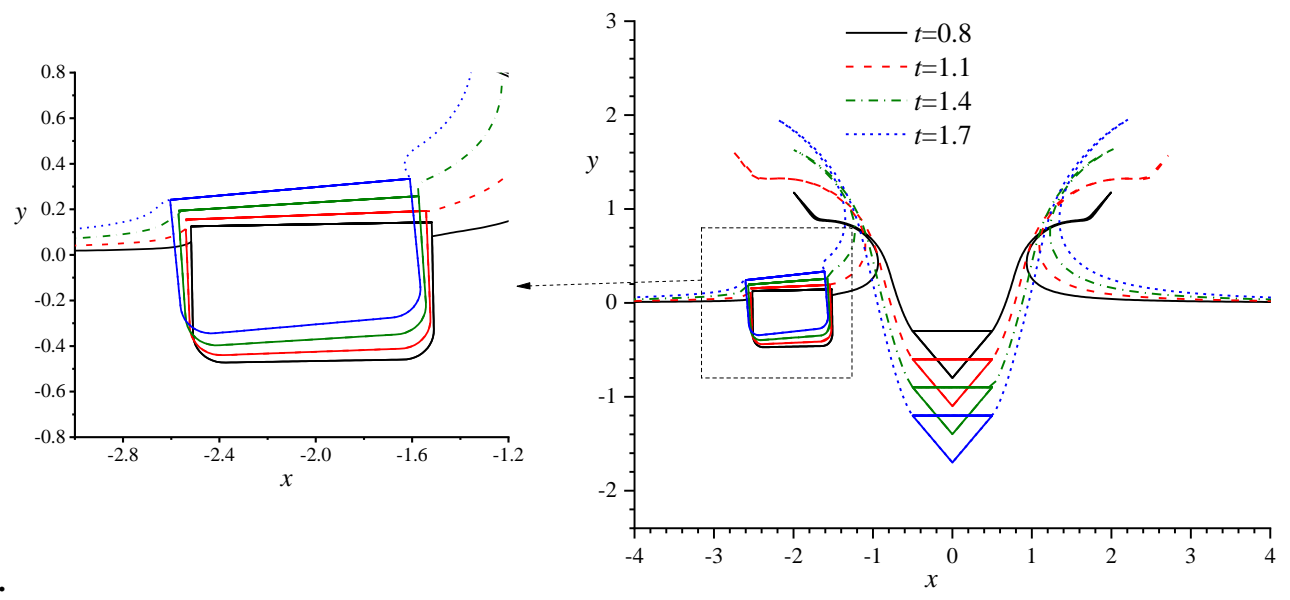

Fig. 7 The free surface profiles at different time instants. (a) $t=0.2$ to $t=0.5$, (b) $t=0.8$ to $t=1.7$ when the symmetrical wedge $\left(\gamma_{1}=\gamma_{2}=45^{\circ}, H_{0}=1, F r=3\right)$ vertically enters water next a freely floating body. 

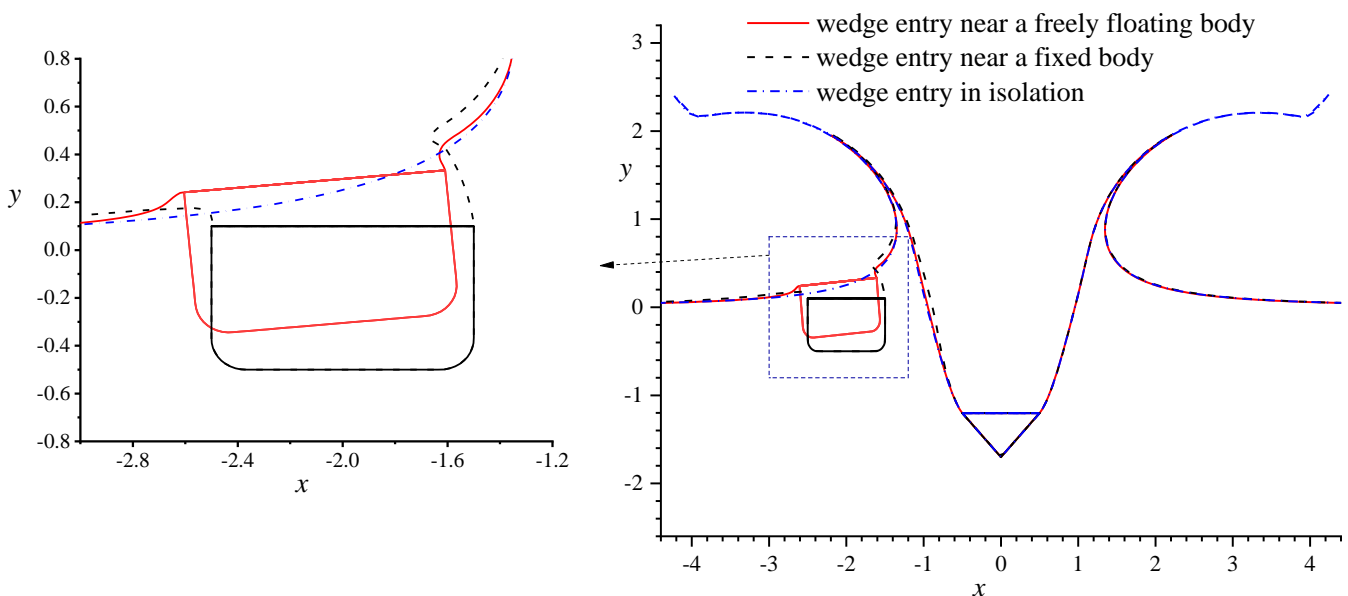

Fig. 8 The free surface profiles at $t=1.7$

Fig.9 shows the free surface profiles and the pressure distributions on the floating body $P_{F}$ at different time instants. $L_{P}$ in the figure is the arc distance measured from the centre point of the bottom plate of the body, and it is positive on the right-hand side of the centre point. When the width of the floating body is 1 , $L_{P}= \pm 0.5$ corresponds to the arc transitional region, $-0.5<L_{P}<0.5$ to the bottom plate and, $L_{P}<-0.5$ to the left plate and $L_{P}>0.5$ to the right plate. In the figure, it can be seen that $P_{F}$ increases with time, and then an upward force is developed. $P_{F}$ on the right-hand side of the floating body is higher than that on the left-hand side, which leads to a negative horizontal force as well as an anti-clockwise moment, as the gravity centre of the floating body is relatively low. Due to these forces and moment, the floating body moves up and from the wedge, as well as rotates in anti-clockwise direction. When $t \geq 0.8, P_{F}$ reduces and its reduction on the righthand side of the floating body is more obvious that that on the left-hand side, especially on the arc transition area on the right-hand side, as shown in Fig.9. Thus, the forces and the moment on the floating body gradually decrease. This can be reflected by the velocity curves of the floating body in three modes shown in Fig.10, in which the acceleration components can be seen through the slope of the curves, respectively. The floating body moves upwards but its vertical velocity is negative as shown in Fig.10b, which is due to the fact that the vertical velocity is positive when the body moves downwards, as defined in Fig.1. 


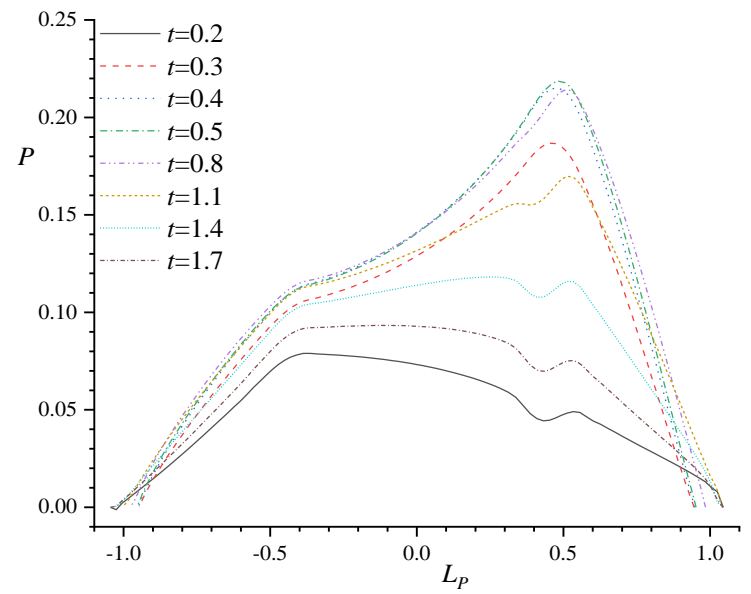

Fig.9 The pressure distribution $P_{F}$ on the floating body surface at different time instants $(t=0.2$ to $t=1.7)$ when the symmetrical wedge $\left(\gamma_{1}=\gamma_{2}=45^{\circ}, H_{0}=1, F r=3\right)$ vertically enters water next a freely floating body.
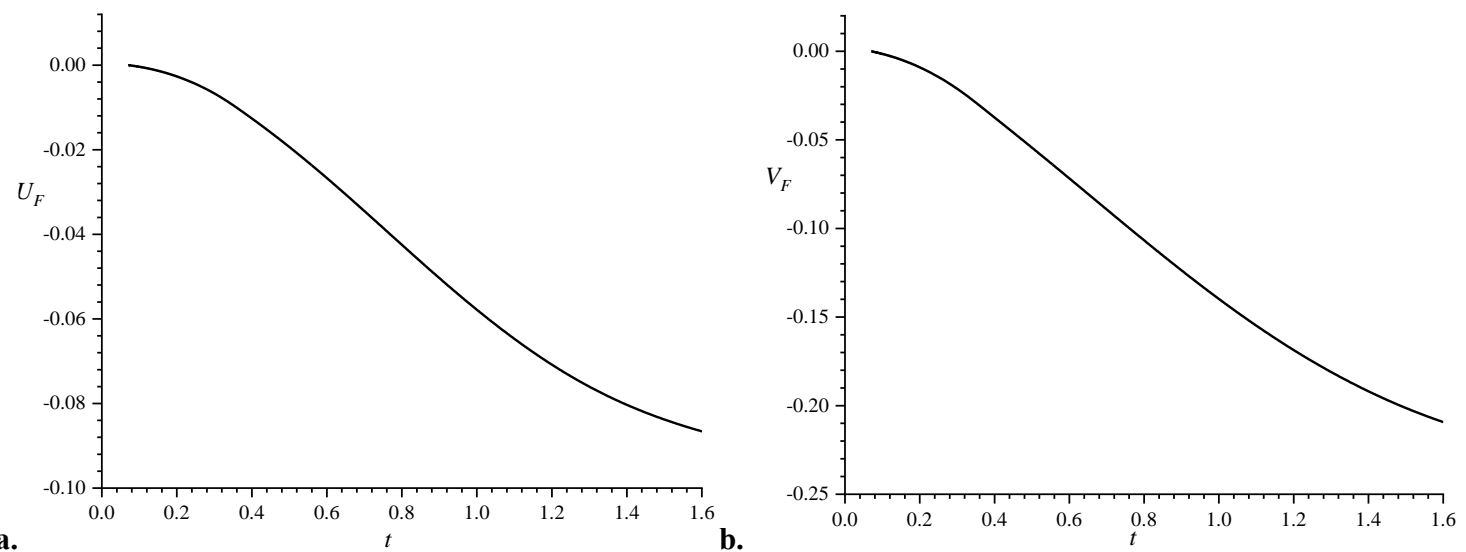

a.

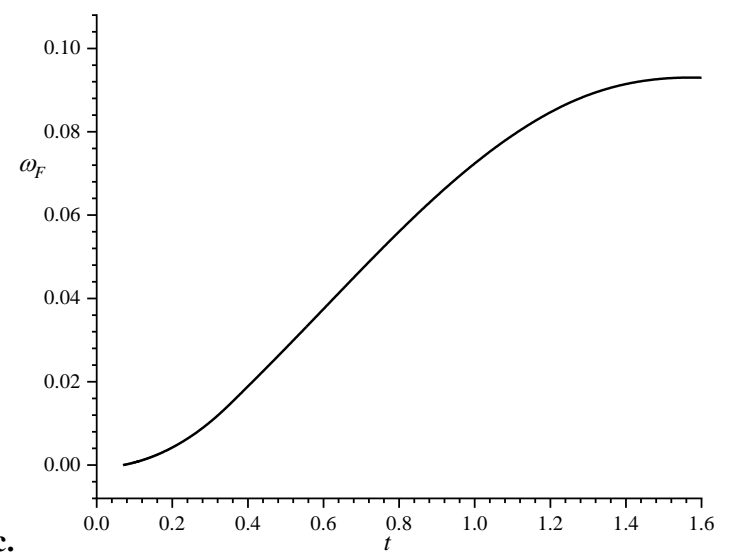

Fig. 10 A symmetrical wedge $\left(\gamma_{1}=\gamma_{2}=45^{\circ}, H_{0}=1, F r=3\right)$ vertically enters water next a freely floating body. (a)horizontal velocity, (b)vertical velocity, (c)rotational velocity of the floating body.

Now we consider the same problem as above but with different initial distances $H_{0}$ between the wedge and the floating body. Fig. 11 shows accelerations and velocities of the floating body in three modes. When the floating body is closer, the disturbance by wedge entry will arrive the body earlier with a bigger force. The floating body will have a larger acceleration. We may notice that the magnitudes of these accelerations reach their peaks around $t=0.4$. This is around the time the jet root detaches from the knuckle of the wedge 
as shown in Fig.7a, beyond which these two bodies also mainly move apart. At larger acceleration, the speed obviously becomes bigger for the same period of time and the body will eventually encounter a larger resistance. Fig. 12 shows the pressure distribution on the floating body $P_{F}$ at $t=0.4$ and 1.6. That can explain the change of accelerations of the floating body. For smaller $H_{0}, P_{F}$ on the right-hand the arc transitional region of the body reduces more rapidly. At smaller $t$, there is a pressure peak in the transition region. At larger $t$, the pressure peak becomes a local trough. Partly this is due to the face that the corner is close the surface of the open cavity behind the wedge. The influence of water entry to the floating body reduces quickly as $H_{0}$ increases. In Fig.11and Fig.12, the difference between accelerations and velocities for $H_{0}=0.5$ and $H_{0}=1.0$ is much larger than that between $H_{0}=1.0$ and $H_{0}=1.5$, and results for $H_{0}=1.5$ is closer to that for $H_{0}=2.0$ than that for $H_{0}=1.0$.

a.

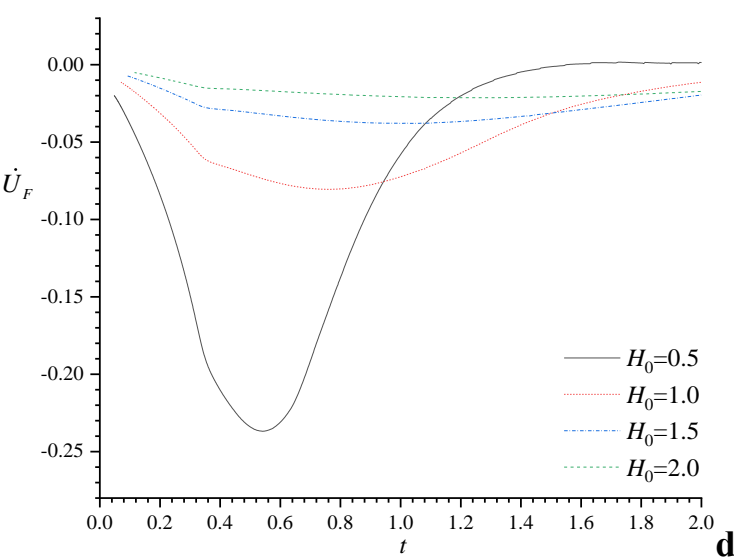

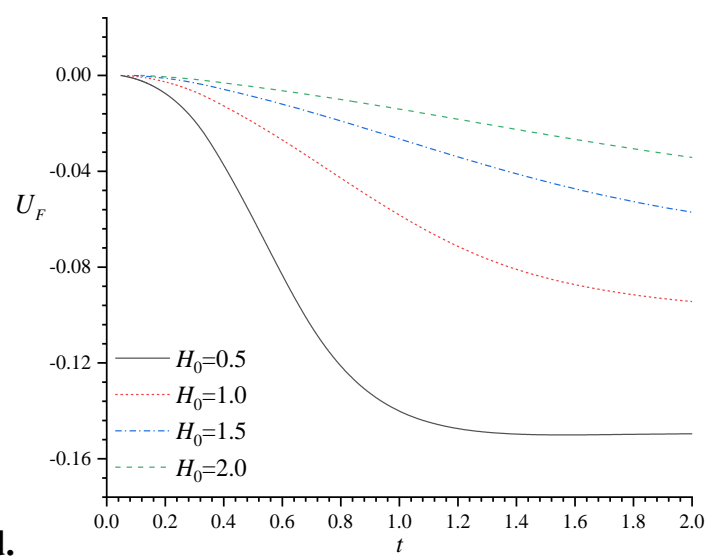

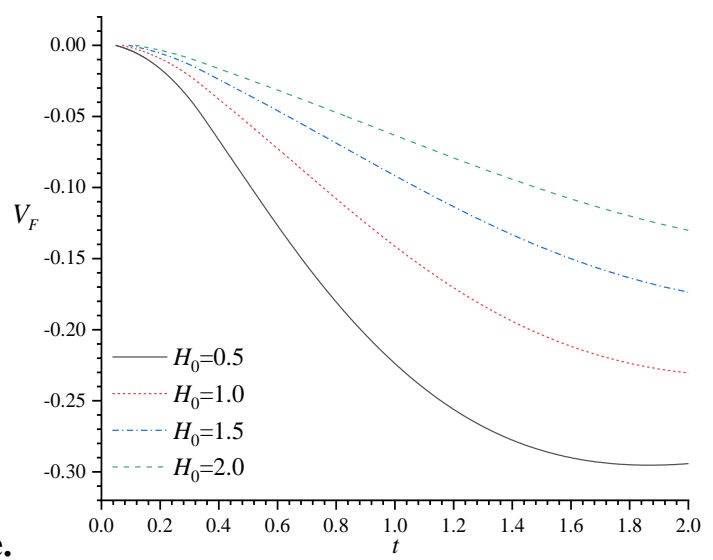



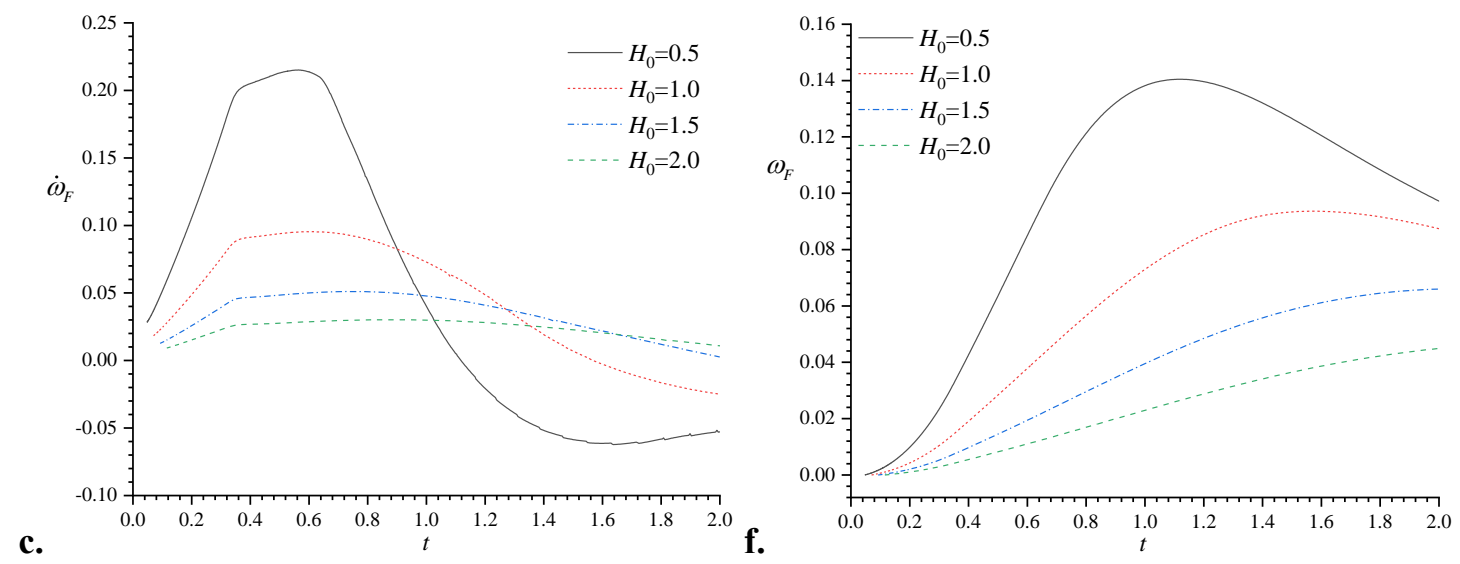

Fig. 11A symmetrical wedge $\left(\gamma_{1}=\gamma_{2}=45^{\circ}, F r=3\right)$ vertically enters water next a freely floating body with different distances $H_{0}$. (a) horizontal acceleration, (b) vertical acceleration, (c) rotational acceleration.

(d) horizontal velocity, (e) vertical velocity, (f) rotational velocity.
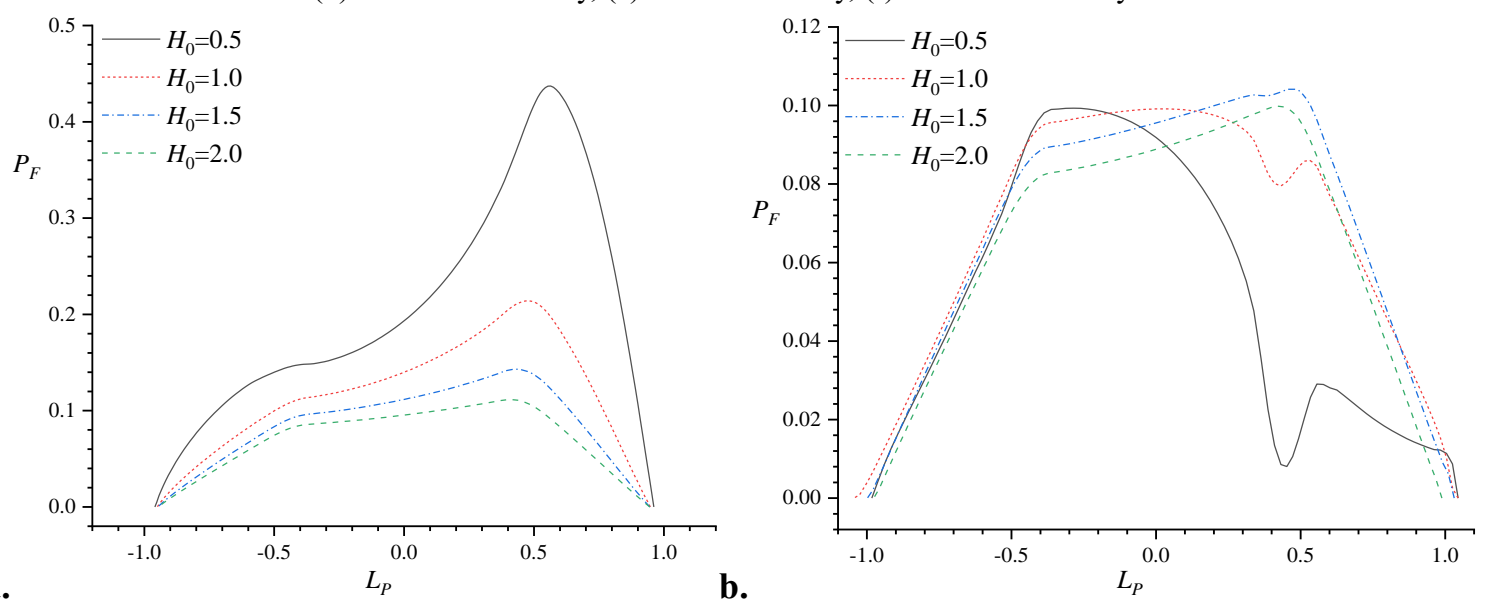

Fig. 12 The pressure distribution on the floating body. (a) $t=0.4$, (a) $t=1.6$

The mass $M_{F}$ of a body has major effect on its motion (Bao et al. [11]). The floating body with larger mass will have larger initial draught $D r$ to achieve the balance between the weight and buoyancy, while other physical parameters are kept the same. The results obtained therefore contain effects from both the body mass $M_{F}$ and body draught $D r$. Fig.13 shows the acceleration and velocity components of the floating body with $M_{F}=0.193,0.293,0.393$ and 0.493 , respectively, and the corresponding initial draught $D r$ are $0.2,0.3,0.4$ and 0.5. Here, as the larger mass corresponds to the larger draught, its right-hand side will receive larger fluid force created by water entry of the wedge. Thus, although the mass is larger, the magnitude of the horizontal acceleration is still larger. At larger draught, the pressure on the bottom of the body is larger as shown in Fig.14. The larger hydrodynamic force counters the larger gravitational force. With its larger mass, the magnitude of the vertical acceleration of the body is smaller, as can be seen from Fig.13b. The wedge obviously creates a larger pressure on the bottom corner on the right-hand side of the body than that on the left, as can be seen from Fig.14. This leads to a positive rotational acceleration as shown in Fig.13c. Similar 
to horizontal mode, the body with larger draught has the larger acceleration, while the rotational inertial is assumed to be the same.

a.

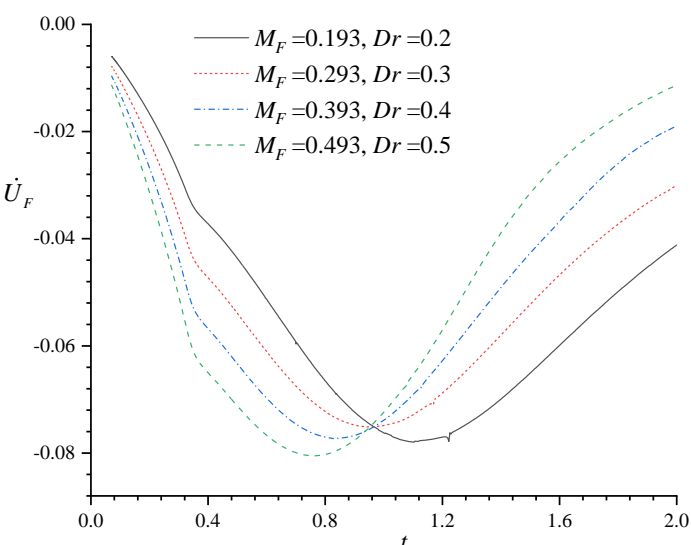

2.0 d.
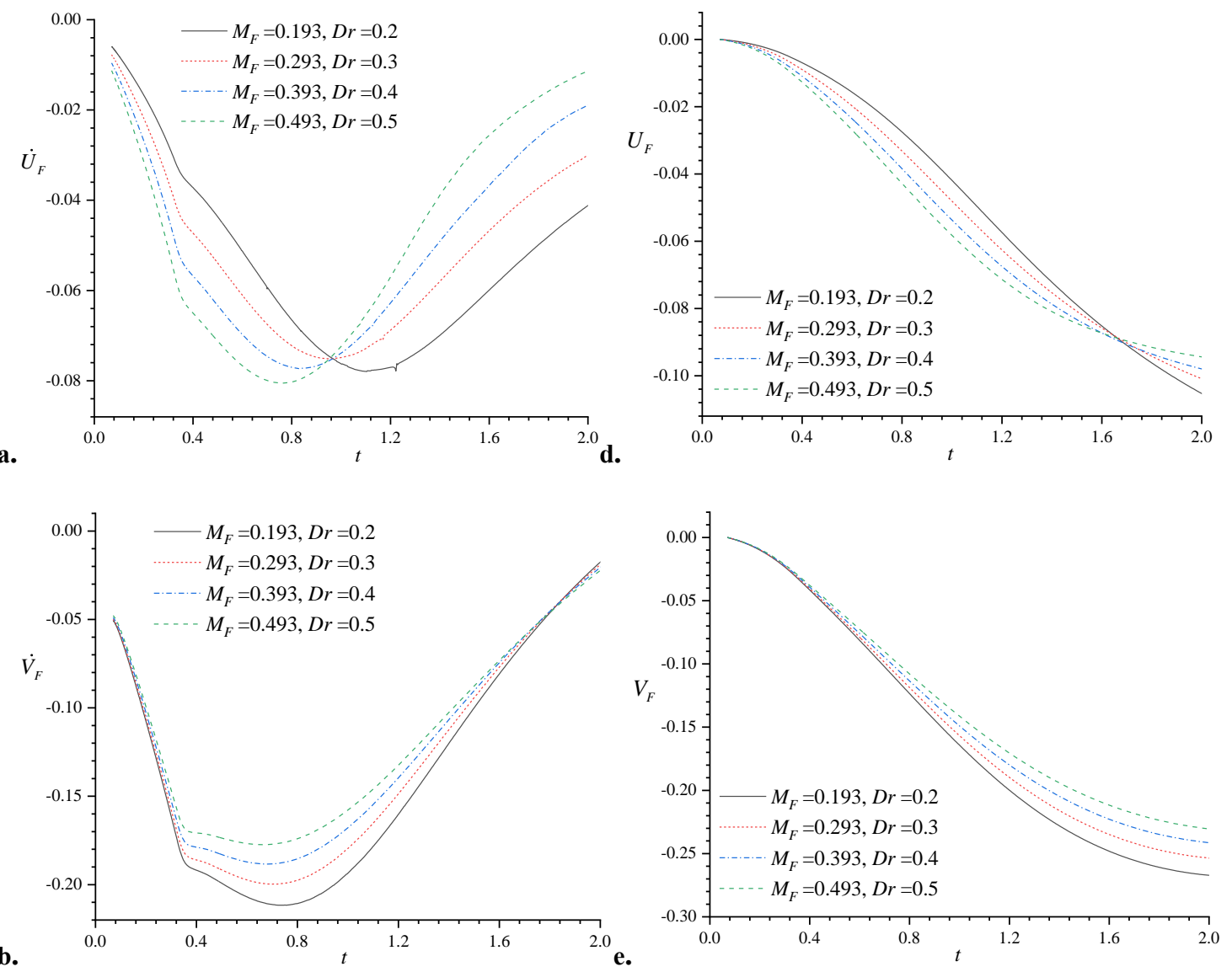

b.

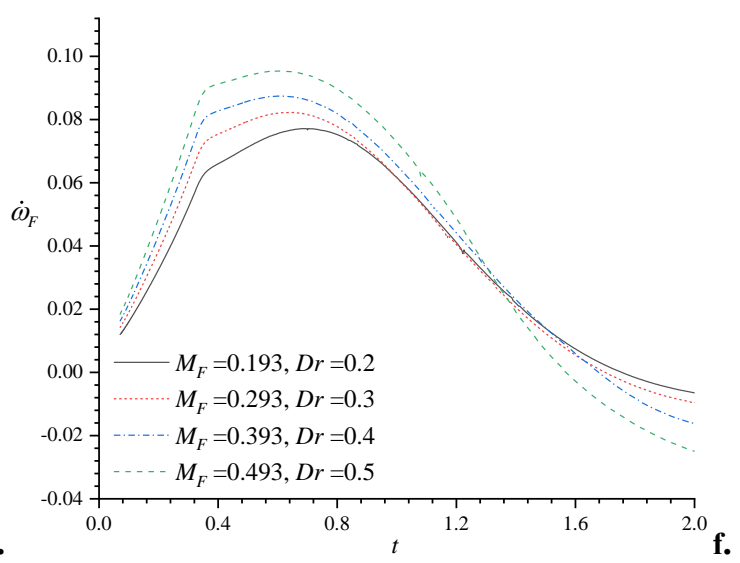

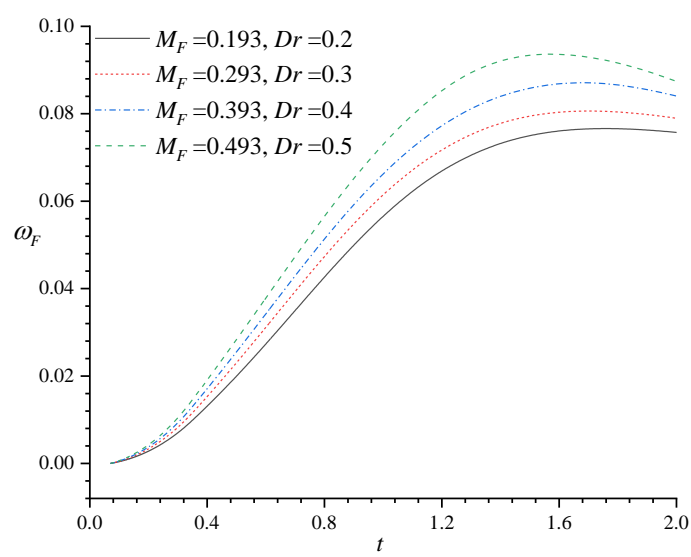

Fig. 13 A symmetrical wedge $\left(\gamma_{1}=\gamma_{2}=45^{\circ}, H_{0}=1, F r=3\right)$ vertically enters water next a freely floating body with different mass $M_{F}$ and initial draught $D r$. (a) horizontal acceleration, (b) vertical acceleration,

(c) rotational acceleration. (d) horizontal velocity, (e) vertical velocity, (f) rotational velocity. 

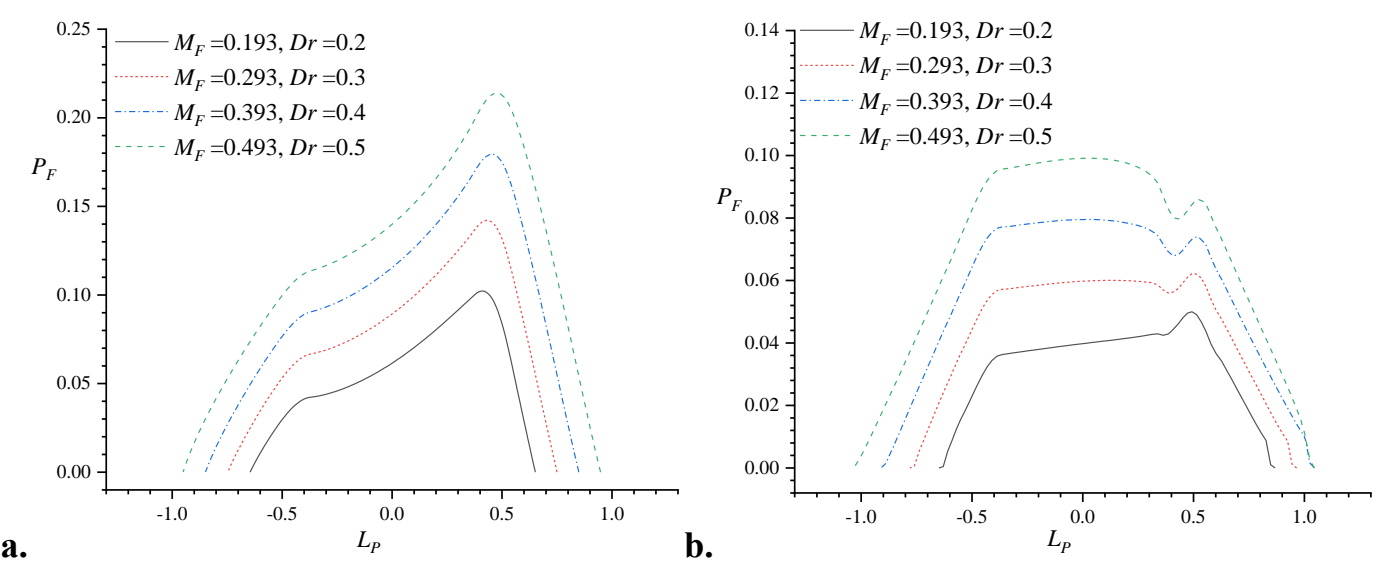

Fig.14 The pressure distribution on the floating body with mass $M_{F}$. (a) $t=0.4$, (a) $t=1.6$.

We then consider wedges with $\gamma_{1}=\gamma_{2}=45^{\circ}$ vertically enter water at a speed of $\mathrm{Fr}_{r}=3$ next the floating body with different ratio of width $B_{F}$ and draught $D r$ while its mass $M_{F}$ remains the same, $H_{0}=1$, and other data of the floating body are shown in Tab.1. Fig.15 shows the accelerations and velocities in three modes of the floating body with $B_{F}=0.9,1.0,1.1$ and 1.2, respectively, and the corresponding initial draught $D r$ are 0.5556 , $0.5,0.4546$ and 0.4167 . Here larger $B_{F}$ means smaller $D r$, which will lead to smaller horizontal force when $H_{0}$ is the same, as shown in Fig.15a. Interestingly, when the $B_{F}$ and $D r$ change, and the initially volume remains the same, the vertical acceleration is nearly the same. This means that although the pressure on the bottom with different width may be different, as shown in Fig.16a, the total force on the bottom may be the same. The difference in the results in the horizontal direction and in rotational direction between various $B_{F}$ is very obvious. The floating body with larger $B_{F}$ will have a smaller magnitude of vertical acceleration in Fig. $15 \mathrm{~b}$ but a larger magnitude of rotational acceleration in Fig.15c. Fig.16a and Fig.16b respectively show the pressure distribution on the floating body $P_{F}$ at $t=0.4$ and $t=1.6$. The body with smaller $B_{F}$ will have larger draught, as well as larger pressure on the body shown in Fig.16a, and thus it will have larger horizontal acceleration as can be seen in Fig.15a. For the body with larger $B_{F}$, the arc transitional region where the peak pressure is located is farther away from the rotation centre, which will lead to a larger amplitude of the rotational accelerations, as shown in Fig.15c. For the same reason, the more rapid decline of the pressure in the arc transition area will result in faster reduction of the magnitude of the rotational acceleration. 

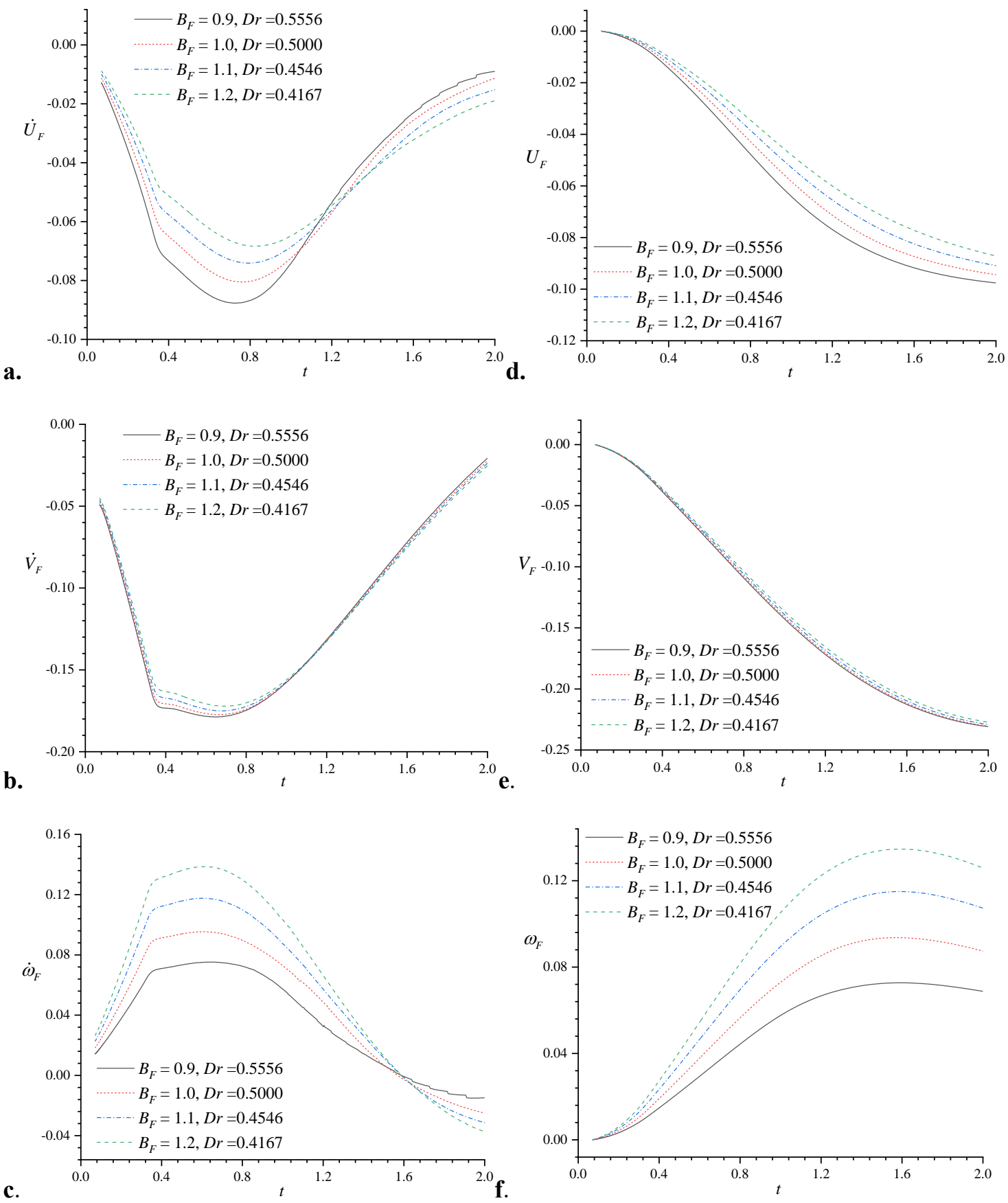

Fig. 15 A symmetrical wedge $\left(\gamma_{1}=\gamma_{2}=45^{\circ}, H_{0}=1, F r=3\right)$ vertically enters water next a freely floating body with different beam and draught ratio. (a) horizontal acceleration, (b) vertical acceleration, (c) rotational acceleration. (d) horizontal velocity, (e) vertical velocity, (f) rotational velocity. 

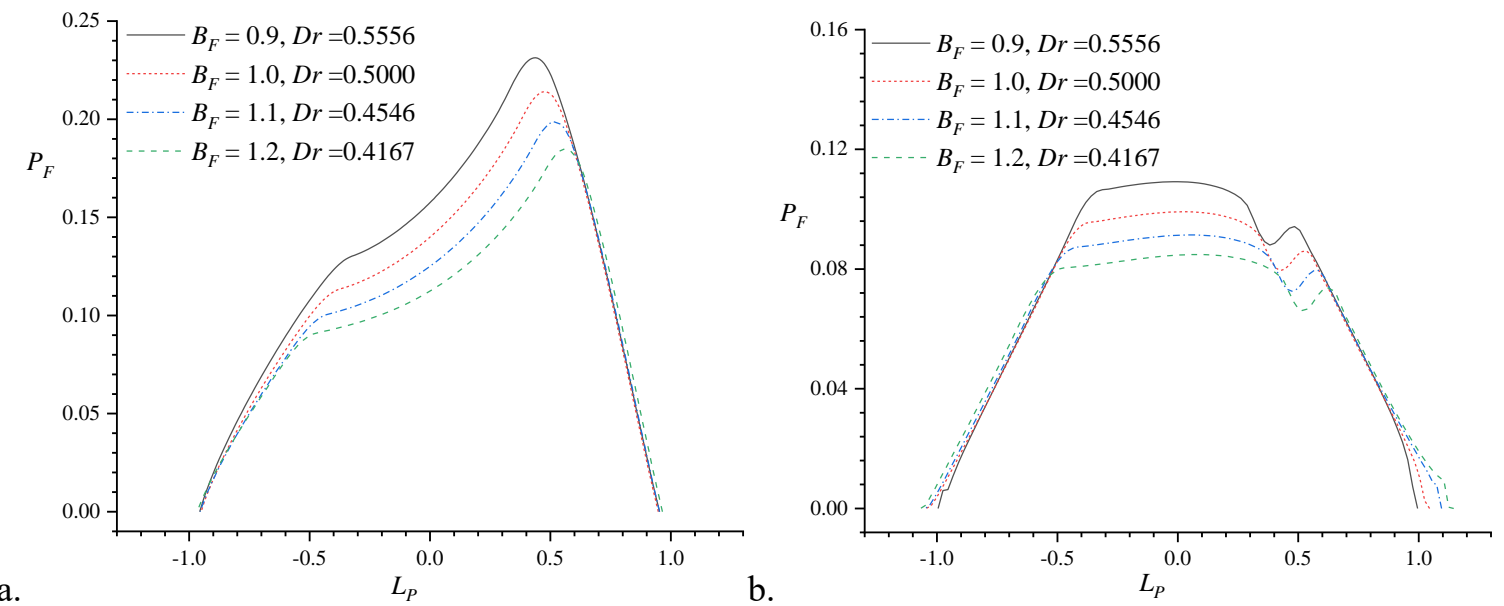

Fig. 16 A symmetrical wedge $\left(\gamma_{1}=\gamma_{2}=45^{\circ}, H_{0}=1, F r=3\right)$ vertically enters water next a freely floating body with different beam and draught ratio. The pressure distribution on the floating body with different $B_{F}$. (a) $t=0.4$, (a) $t=1.6$.

\subsection{Water entry of a wedge in freefall next a floating body}

We consider an asymmetrical wedge with heel angle $\theta_{W 0}=-10^{\circ}\left(\gamma_{1}=35^{\circ}, \gamma_{2}=55^{\circ}\right)$ vertically entering water with $F r_{0}=3$ in freefall next a floating body $\left(H_{0}=0.5\right)$. Fig. 17 gives the accelerations and velocities of the wedge in three modes with and without influence of a floating body. Overall, the influence of the floating body on the wedge is not very significant. At initial stage, the interaction between the wedge and the floating body is negligible, and those curves in each of Fig.17 almost coincide with each other. Later on, influence of the floating body on the wedge in horizontal direction becomes quite obvious. The fixed body has larger influence. It acts like a fixed wall nearby, while the floating body will be pushed away and its effect will accordingly be reduced. Fig. 18 shows the free surface profiles at $t=1.5$. There is no obvious difference in the free surface profiles on the right-hand side of the wedge. Their difference on the left-hand hand side is obvious.

a.
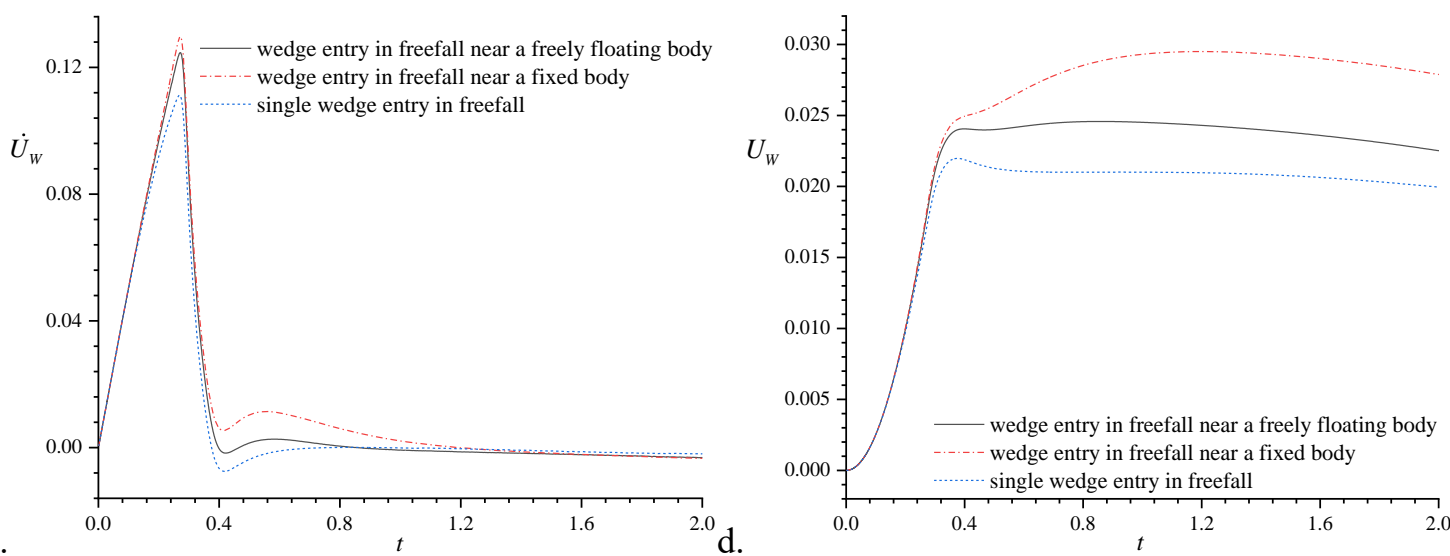

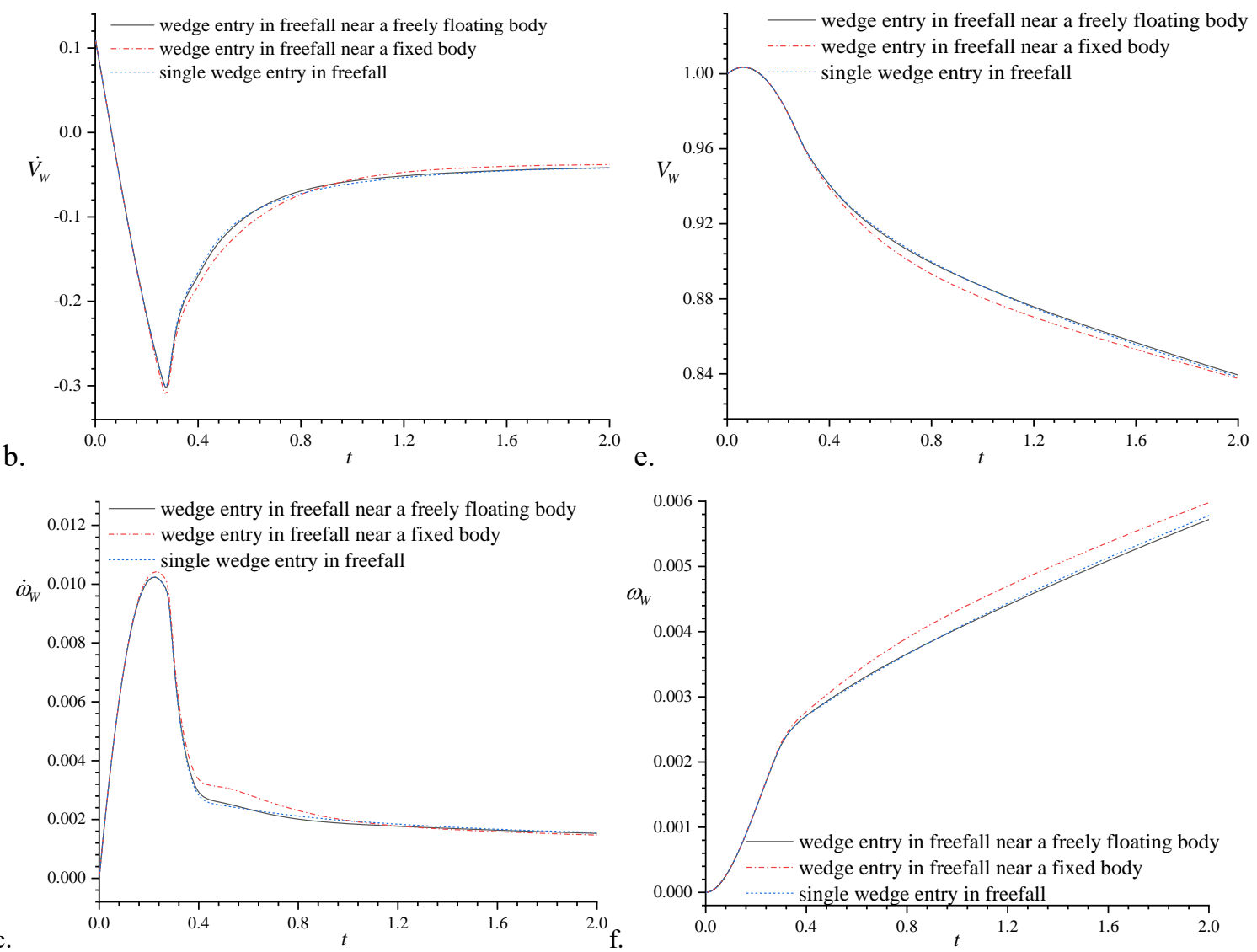

Fig. 17 Comparison between accelerations and between velocities of the wedge: (a) horizontal acceleration,

(b) vertical acceleration, (c)rotational acceleration, (d)horizontal velocity, (e)vertical velocity, (f) rotational velocity.

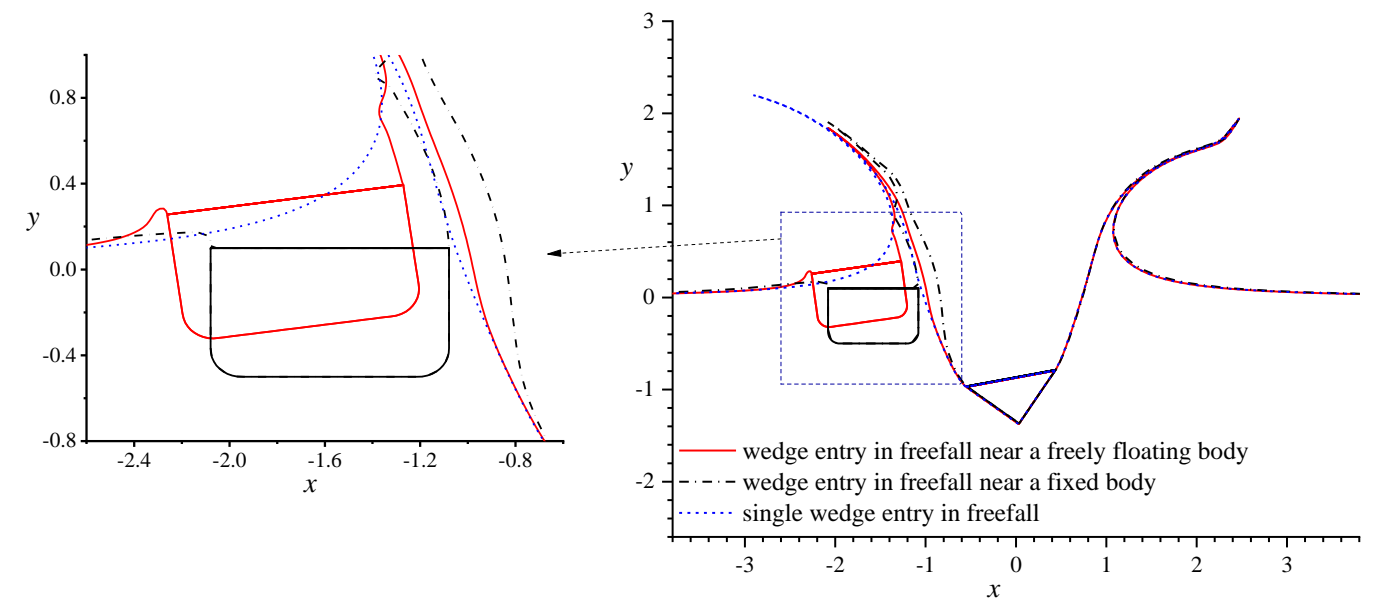

Fig.18 Comparison between the free surface profiles at $t=1.5$.

We then compare the effect of the above wedge on the floating body when the wedges enter water in freefall and at a constant speed, respectively. The velocities of the floating body in three modes are shown in Fig.19. At initial stage, the influence of wedge entry on the floating body is negligible, and thus there is not much difference between the two curves of a wedge at constant speed or in freefall. The magnitudes of the former are larger than that of the latter as time increases. The reason is that the vertical velocity of the wedge 
entering in freefall decreases, and at the same time the wedge is pushed away from the floating body as shown in Fig.17d. Its effect on the floating body then decreases.

a.
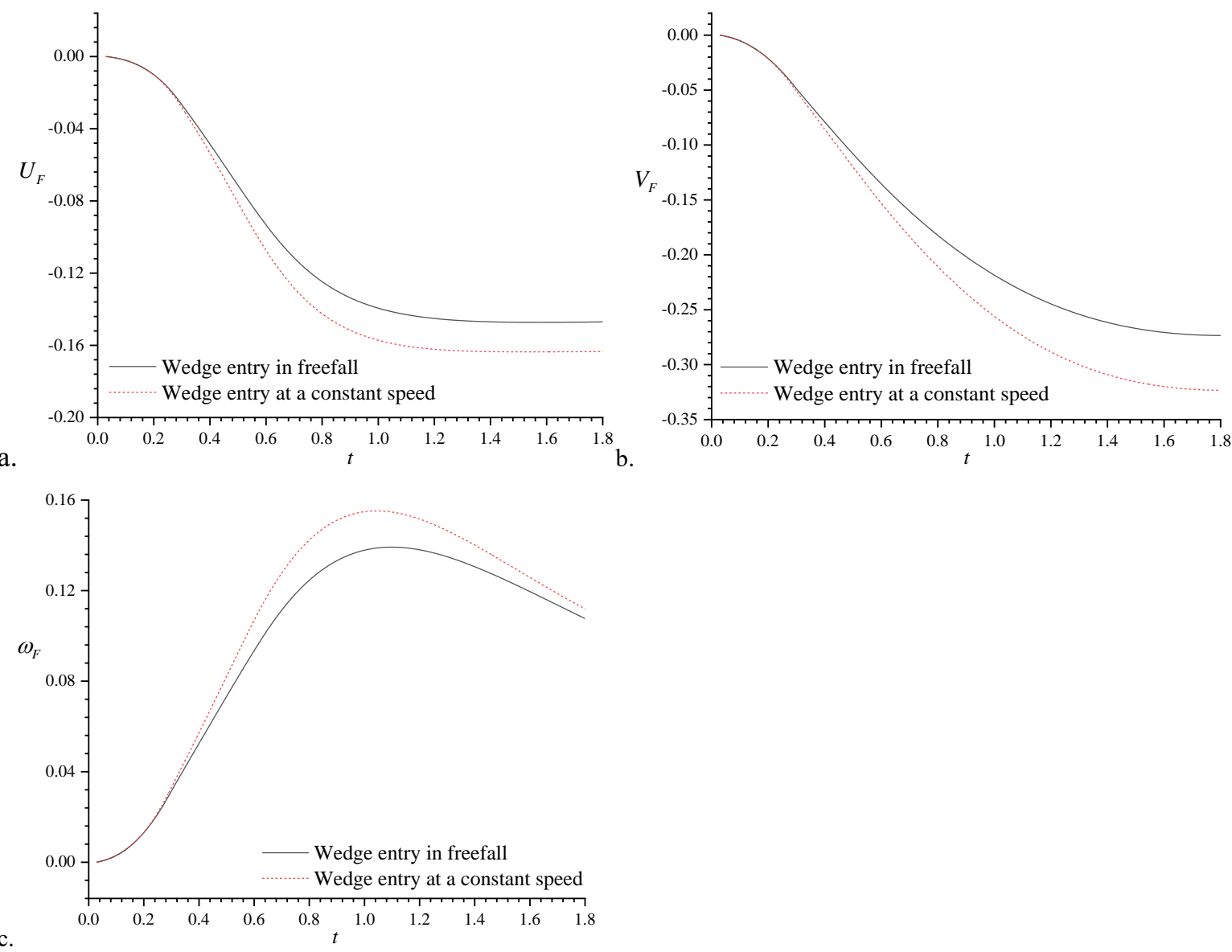

Fig.19 The velocities of the floating body due to water entry of a wedge nearby:(a) horizontal velocity,

(b) vertical velocity, (c) rotational velocity. The solid line: freefall, the dashed line: constant speed.

We now consider wedges with different $\theta_{W 0}$ vertically enter water in freefall next a freely floating body. When $\theta_{W 0}<0^{\circ}$, the left deadrise angle $\gamma_{1}$ is smaller and the right deadrise angle $\gamma_{2}$ is larger, as can be seen from Eq.(47). Fig.20 shows the accelerations and velocities of the floating body in three modes. The difference between the accelerations of different $\theta_{W 0}$ increases first, because smaller $\gamma_{1}$ of the wedge gives a larger force to the floating body although its tip is farther away from the body when $H_{0}$ remains to be the same. At the same time, the smaller $\gamma_{1}$ will give a larger pressure distribution on the corresponding side of the wedge, which will in turn increase $\gamma_{1}$, and subsequently reduce the difference between the accelerations. Fig.21 shows the free surface profiles at $t=0.4$, at which the jet root has detached from the knuckle. The force on the wedge with smaller deadrise angle is larger and will decrease more after the jet root has detached from the body, as observed in (Bao et al. [11]). In Fig.20, the magnitudes 
of vertical and rotational accelerations of the floating body decrease after about $t=0.4$.

a.

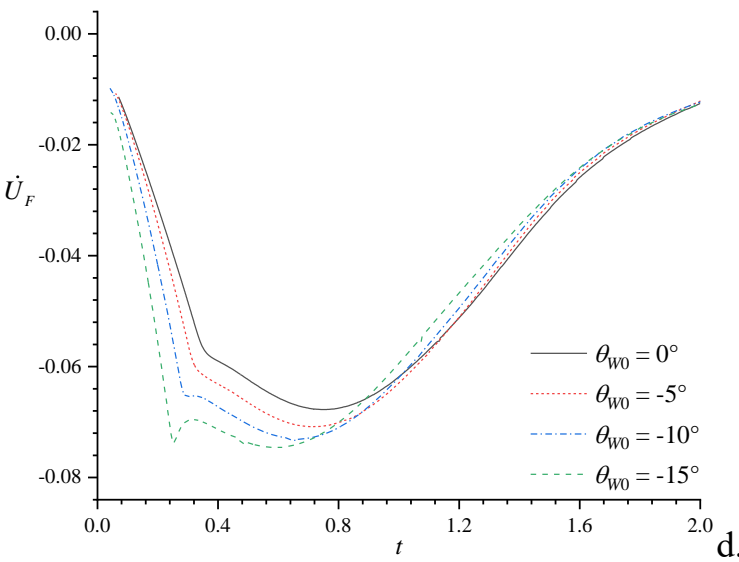

b.
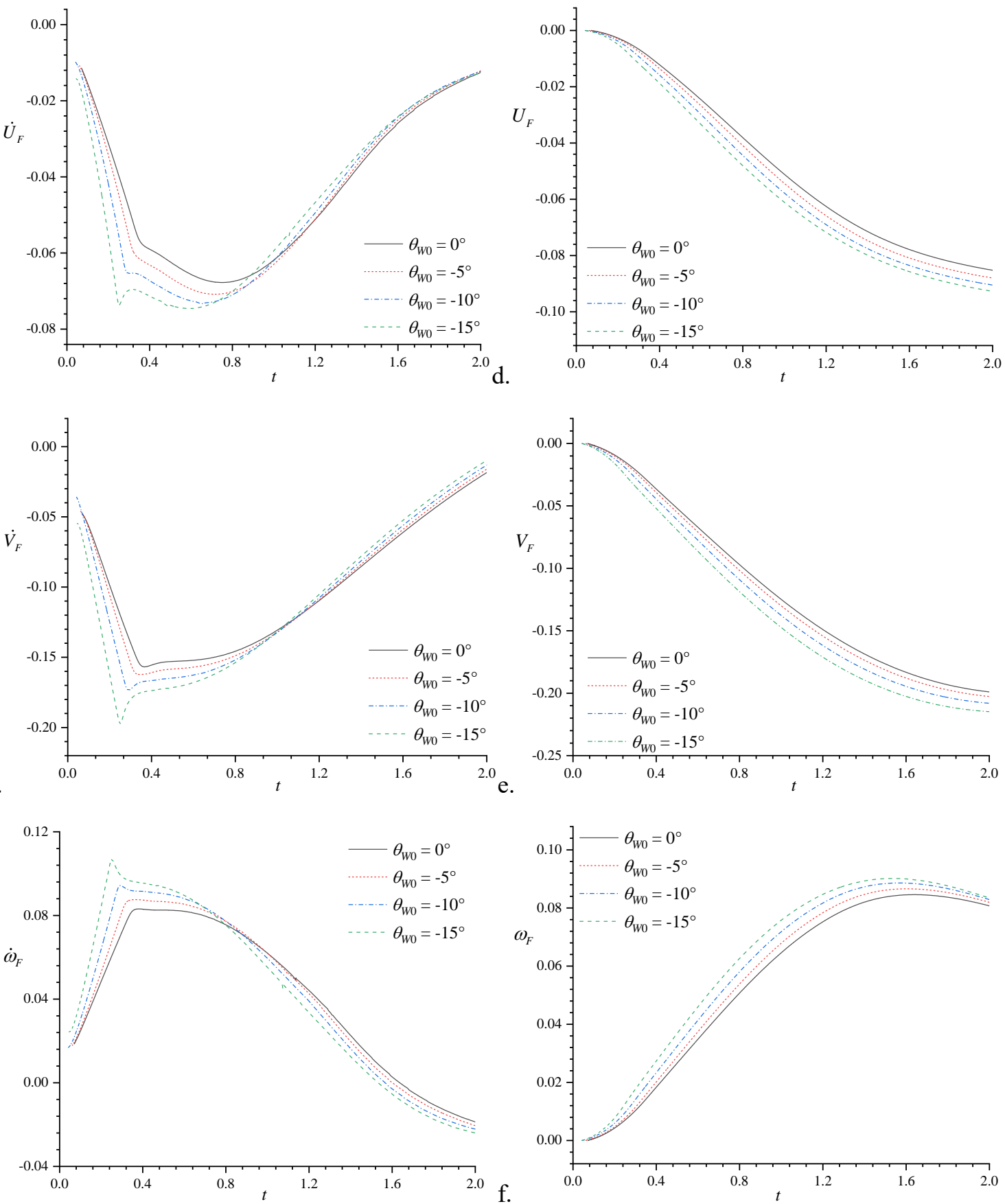

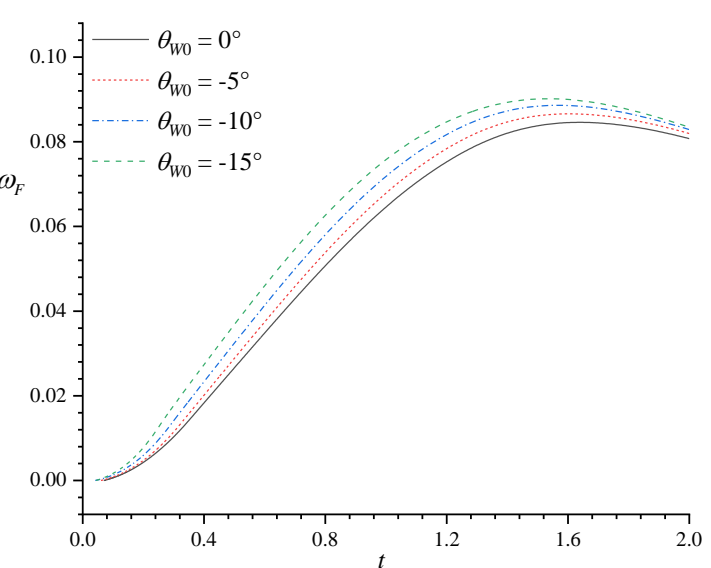

Fig.20 The accelerations and velocities of a freely floating body due to vertical entry $\left(F r_{0}=3, H_{0}=1\right)$ of a wedge $\left(\gamma=45^{\circ}\right)$ with different $\theta_{W 0}$. (a) horizontal acceleration, (b) vertical acceleration, (c) rotational acceleration.

(c) horizontal velocity, (d) vertical velocity, (e) rotational velocity. 


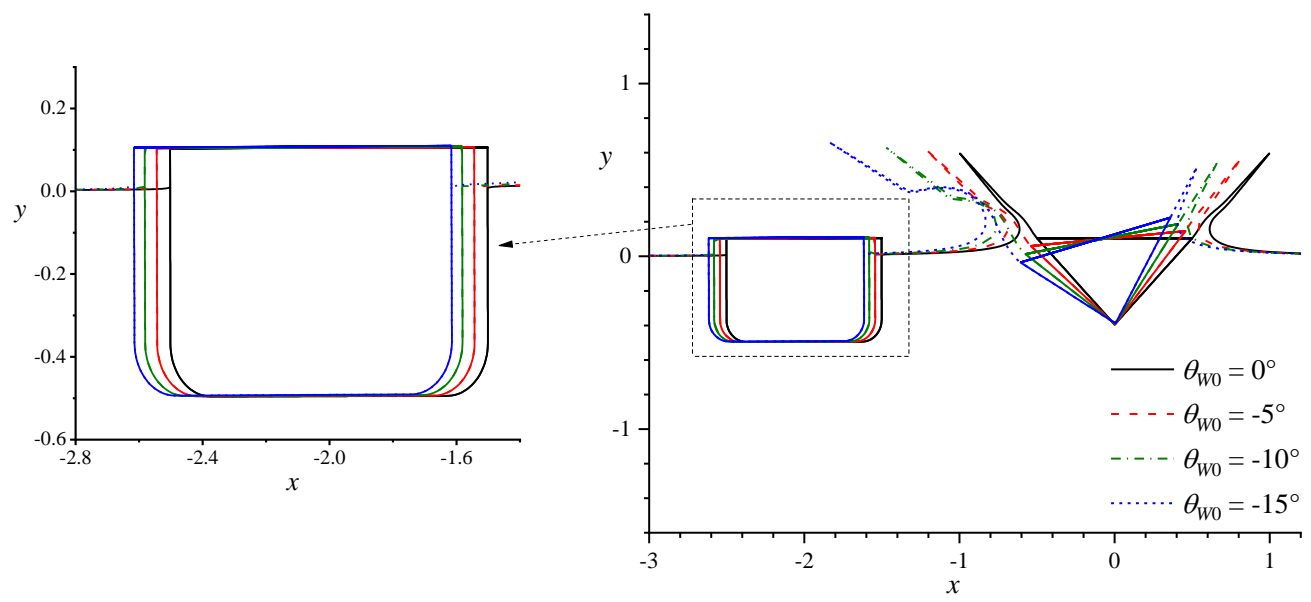

Fig. 21 The free surface profiles at $t=0.4$ due to vertical entry $\left(F r_{0}=3, H_{0}=1\right)$ of a wedge $\left(\gamma=45^{\circ}\right)$

with different $\theta_{W 0}$ near a floating body

To study the behaviour at oblique entry, we consider the wedge entering water in freefall with different horizontal velocities $U_{W 0}=0.3,0,-0.1$ and -0.3 . Fig. 22 shows the accelerations and velocities in three modes of the floating body. When $U_{W 0}$ is negative, the wedge moves towards the body when it goes down, and the distance between the wedge and the floating body decreases. In this case, the difference between the accelerations at different $U_{W 0}$ remains to be obvious over the most period of the simulation. This is because the flow near the body will be greatly affected by the horizontal velocity of the wedge $U_{W}$. At the same time, the distance between the wedge and the floating body keeps changing. Consequently, the difference between the accelerations remains to be large.

a.

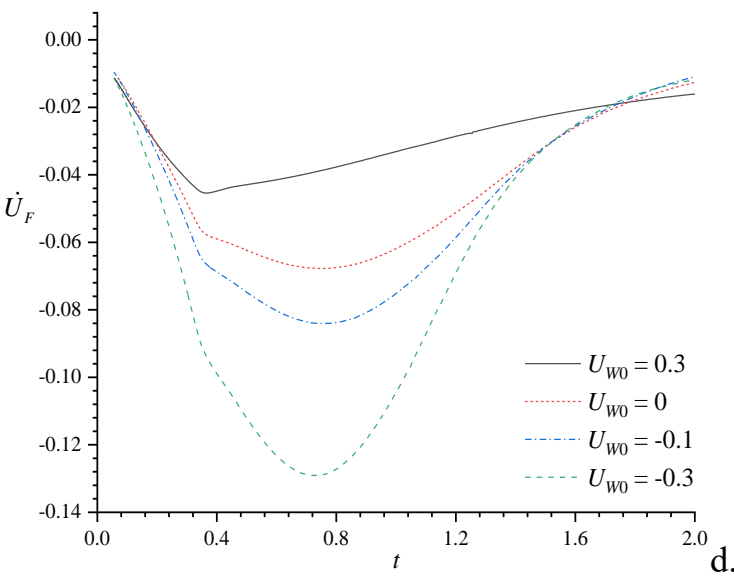

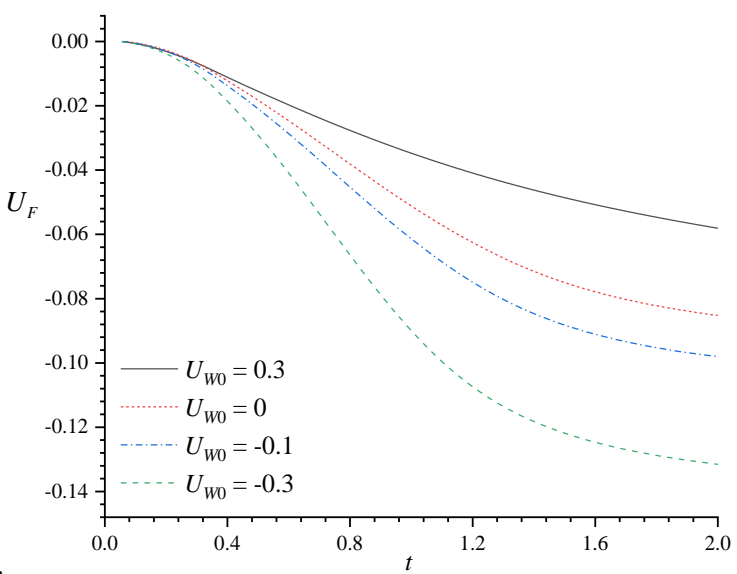



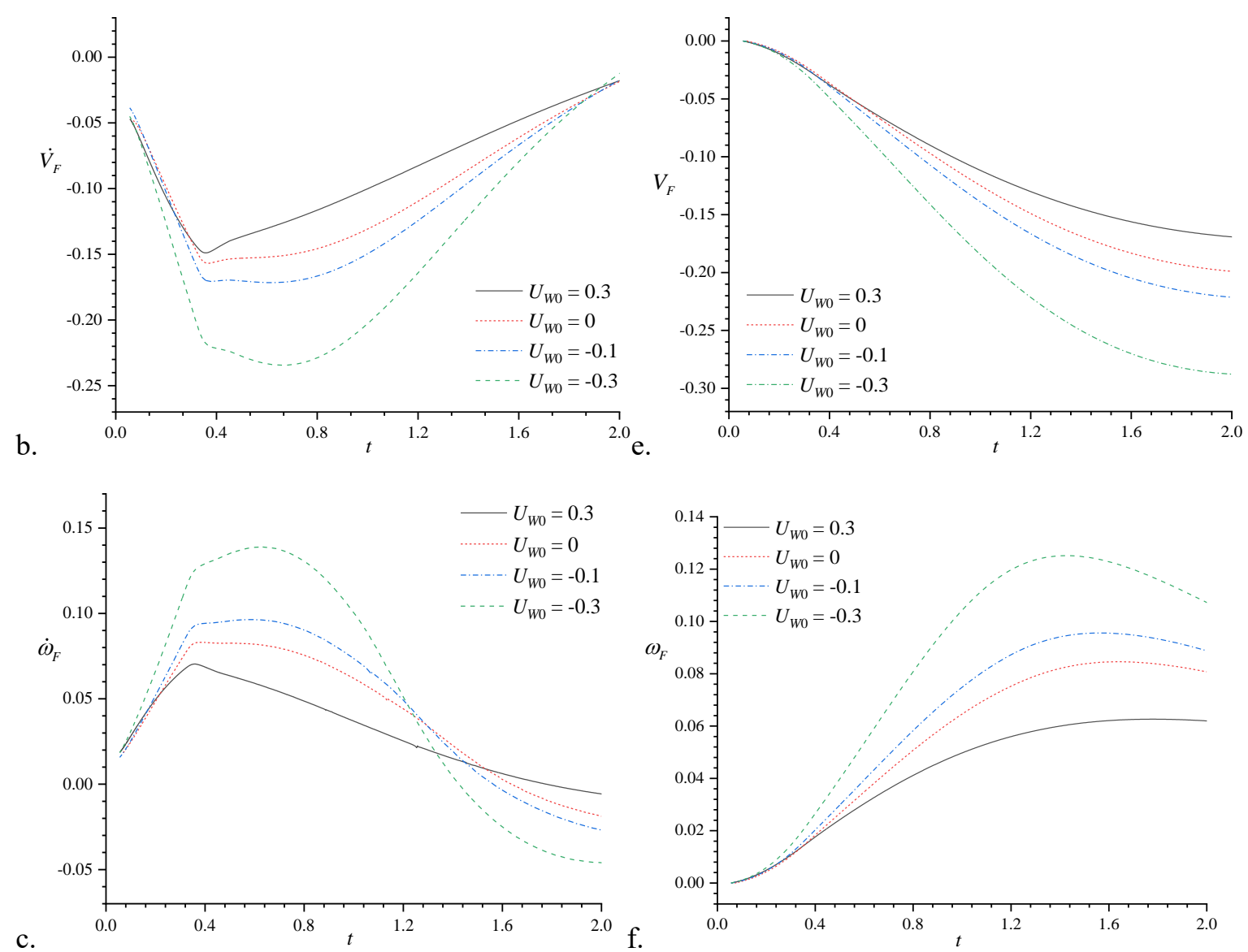

Fig. 22 Accelerations and velocities of a freely floating body due to oblique entry $\left(F r_{0}=3, H_{0}=1\right)$ of a wedge $\left(\gamma_{1}=\gamma_{2}=45^{\circ}\right)$ at different $U_{W 0}$. (a) horizontal acceleration, (b) vertical acceleration, (c) rotational acceleration.

(c) horizontal velocity, (d) vertical velocity, (e) rotational velocity.

Finally, we consider a symmetrical wedge enters water with different initial rotational velocities $\omega_{w 0}$. If $\omega_{W}$ is positive, the flow on the left-hand side of the wedge pushed by entry is enhanced. Fig.22 shows the accelerations and velocities in three modes. As the wedge enters water, the wetted surface on the left-hand side of the wedge increases and at the same time the left deadrise angle $\gamma_{1}$ of the wedge decreases. That makes the difference between the accelerations in Fig.23 more obvious as time increases. Fig.24 shows the free surface profiles at $t=0.7$. Due to different $\omega_{W 0}$, the deadrise angles of the wedge are obviously different, as well as the free surface shape around the wedge. However, there is little difference in the free surface shapes near the floating body. 

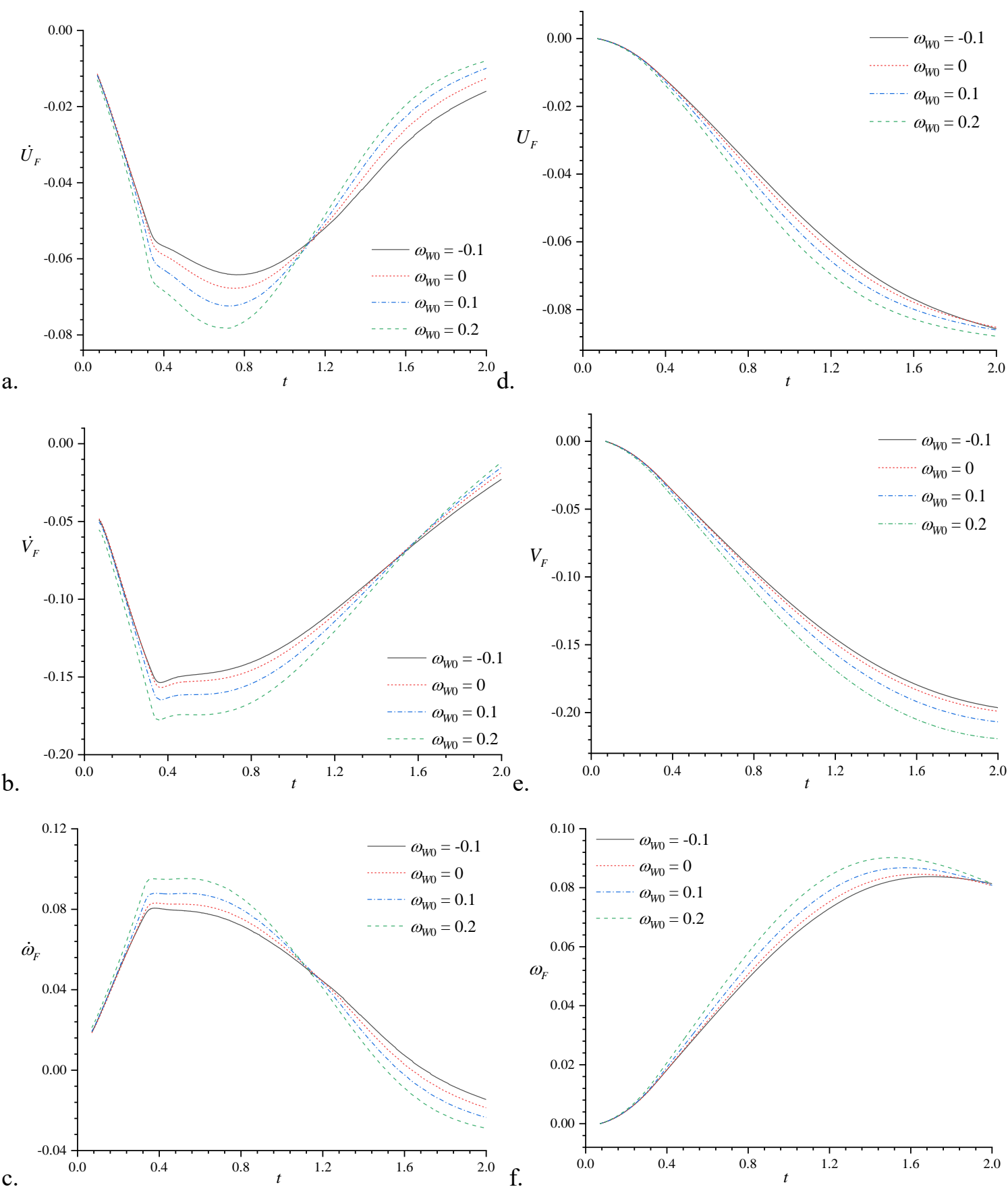

Fig.23 Accelerations and velocities of a freely floating body due to vertical entry $\left(F r_{0}=3, H_{0}=1\right)$ of a wedge $\left(\gamma_{1}=\gamma_{2}=45^{\circ}\right)$ nearby at different $\omega_{w 0}$. (a) horizontal acceleration, (b) vertical acceleration, (c) rotational acceleration.

(d) horizontal velocity, (e) vertical velocity, (f) rotational velocity. 


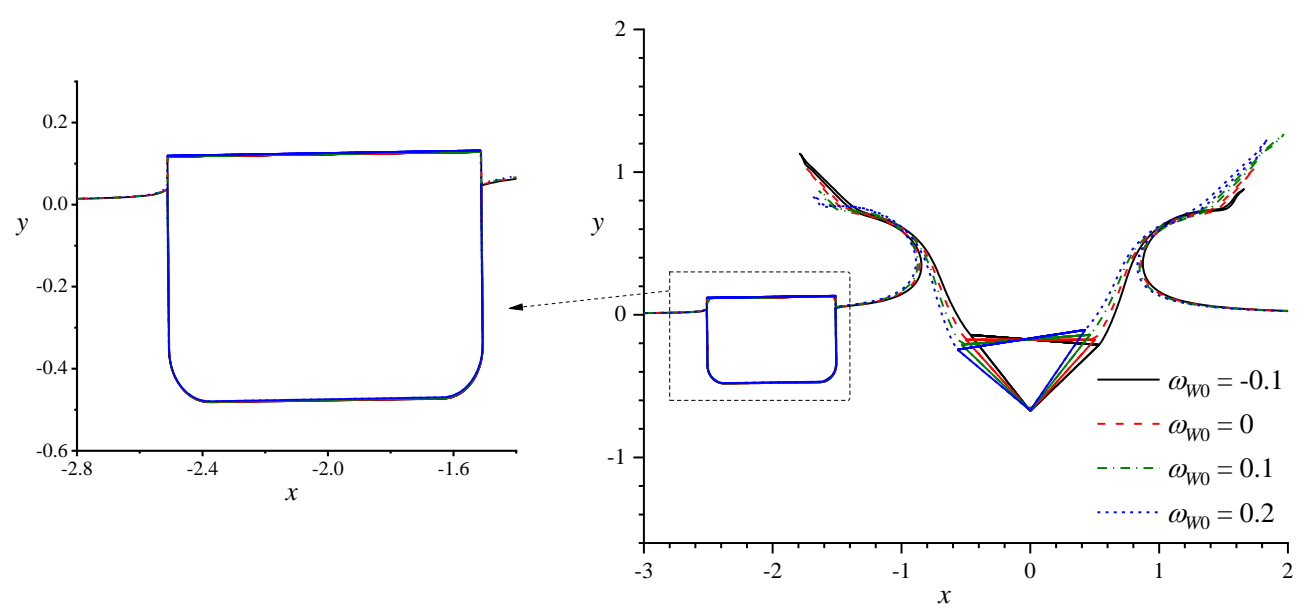

Fig.24 Free surface profiles at $t=0.7$ due to vertical entry $\left(F r_{0}=3, H_{0}=1\right)$ of a wedge $\left(\gamma_{1}=\gamma_{2}=45^{\circ}\right)$ at different $\omega_{w 0}$ near a floating body

\section{Conclusions}

The problem of a finite wedge entering water near a floating body is solved by using the boundary element method based on the velocity potential flow theory. The simulation through time stepping is divided into two phases, depending on whether the fluid around the freely floating body is significantly disturbed by water entry of the wedge. The nonlinear mutual dependence of the motion of the floating body, the wedge motion and the fluid flow is decoupled by the auxiliary function method. Through these obtained results, we can draw the following conclusions.

(1) At initial stage, the effect on the floating body by the wedge is small. It increases rapidly as the wedge moves down into water. Large pressure on the body will be created, especially near its right bottom. After the root of the jet on the wedge detaches from its knuckle as the wedge moves further down, the effect of the wedge on the floating body decreases rapidly. The overall effect of the floating body on the wedge itself is relatively small.

(2) The effect of the wedge on the floating body decreases rapidly with the horizontal distance between them. When the distance is small, the large peak pressure can occur around its bottom right corner at earlier stage. However, this peak becomes a trough at later stage, partly because the corner is close to the surface of open cavity behind the wedge.

(3) When the body mass increases, its initial draught also has to increase to maintain the static hydrostatic balance, if the beam of the body remains the same. The body then receives a larger horizontal force caused by the wedge, and responds with a larger magnitude of horizontal acceleration even it is heavier. 
However, the magnitude of the vertical acceleration is smaller. If the body mass is fixed, when the beam changes, its draught also has to change to maintain the volume. In such a case, the vertical acceleration is not sensitive to the change of the beam while for the magnitude of the horizontal acceleration is larger for smaller beam with larger draught.

(4) When the wedge enters water obliquely, it will have a much larger effect on the floating body, mainly due to the face the distance between them changes, as well as the change of the direction of the flow velocity relative to the wedge surface on the body side. When the wedge enters the water with a rotational angle, it will also have major effect on the body, mainly due to the change of the deadrise angle of the wedge on the body side.

\section{Acknowledgements}

This work is supported by Lloyd's Register Foundation through the joint center involving University College London, Shanghai Jiao-tong University and Harbin Engineering University, to which the authors are most grateful. Lloyd's Register Foundation helps to protect life and property by supporting engineeringrelated education, public engagement and the application of research.

This work is also supported by the National Natural Science Foundation of China (Grants number 51879123)

\section{References}

[1] Dobrovol'Skaya, Z. N. (1969). On some problems of similarity flow of fluid with a free surface. Journal of Fluid Mechanics, 36(4), 805-829.

[2] Zhao, R., \& Faltinsen, O. (1993). Water entry of two-dimensional bodies. Journal of Fluid Mechanics, $246,593-612$.

[3] Wu, G. X., Sun, H., \& He, Y. S. (2004). Numerical simulation and experimental study of water entry of a wedge in free fall motion. Journal of Fluids and Structures, 19(3), 277-289.

[4] Xu, G. D., Duan, W. Y., \& Wu, G. X. (2010). Simulation of water entry of a wedge through free fall in three degrees of freedom. In Proceedings of the Royal Society of London A: Mathematical, Physical and Engineering Sciences (Vol. 466, No. 2120, pp. 2219-2239). The Royal Society.

[5] Semenov, Y. A., \& Iafrati, A. (2006). On the nonlinear water entry problem of asymmetric wedges. 
Journal of Fluid Mechanics, 547, 231-256.

[6] Zhao, R., Faltinsen, O., \& Aarsnes, J. (1996). Water entry of arbitrary two-dimensional sections with and without flow separation. In Proceedings of the 21 st symposium on naval hydrodynamics (pp. 408423). Trondheim, Norway, National Academy Press, Washington, DC, USA.

[7] Iafrati, A., \& Battistin, D. (2003). Hydrodynamics of water entry in presence of flow separation from chines. In The 8th International Conference on Numerical Ship Hydrodynamics (pp. 22-25).

[8] Sun, H., \& Faltinsen, O. M. (2009). Water entry of a bow-flare ship section with roll angle. Journal of marine science and technology, 14(1), 69-79.

[9] Wang, J., \& Faltinsen, O. M. (2013). Numerical investigation of air cavity formation during the highspeed vertical water entry of wedges. Journal of Offshore Mechanics and Arctic Engineering, 135(1), 011101.

[10] Bao, C. M., Wu, G. X., \& Xu, G. D. (2016). Simulation of water entry of a two-dimension finite wedge with flow detachment. Journal of Fluids and Structures, 65, 44-59.

[11] Bao, C. M., Wu, G. X., \& Xu, G. (2017). Simulation of freefall water entry of a finite wedge with flow detachment. Applied Ocean Research, 65, 262-278.

[12] Wu, G. X. (2006). Numerical simulation of water entry of twin wedges. Journal of Fluids and Structures, 22(1), 99-108.

[13] Xu, G. D., Duan, W. Y., \& Wu, G. X. (2009). Time domain simulation of water entry of twin wedges through free fall motion. Proceedings of 24th International workshop on water waves and floating bodies, St, Petersburg, Russia.

[14] Maki K. J., Ye H., Khabakhpasheva T. I., Korobkin A.A. (2017). Impact of a Wedge-Shaped Body with Influence of Broken Ice. Proceedings of the 32th International Workshop on Water Waves and Floating Bodies, Dalian, China.

[15] Wu, G. X., \& Eatock Taylor, R. (2003). The coupled finite element and boundary element analysis of nonlinear interactions between waves and bodies. Ocean Engineering, 30(3), 387-400.

[16] Lu, C. H., He, Y. S., \& Wu, G. X. (2000). Coupled analysis of nonlinear interaction between fluid and structure during impact. Journal of fluids and structures, 14(1), 127-146.

[17] Wu, G. X. (2007). Two-dimensional liquid column and liquid droplet impact on a solid wedge. Quarterly Journal of Mechanics and Applied Mathematics, 60(4), 497-511. 
[18] Wu G X. (1998). Hydrodynamic force on a rigid body during impact with liquid. Journal of Fluids and Structures, 12(5): 549-559.

[19] Xu, G. D., \& Wu, G. X. (2013). Hydrodynamics of a submerged hydrofoil advancing in waves. Applied Ocean Research, 42, 70-78.

[20] Koo, W., \& Kim, M. H. (2004). Freely floating-body simulation by a 2D fully nonlinear numerical wave tank. Ocean Engineering, 31(16), 2011-2046.

[21] Wang, J., Sun, S. L., \& Hu, J. (2017). The coupling analysis of tank motion and sloshing by a fully nonlinear decoupling method. Nonlinear Dynamics, 89(2), 971-985. 